\title{
Bayesian Identification of Mean-Field Homogenization model parameters and uncertain matrix behavior in non-aligned short fiber composites
}

\author{
Mohamed Mohamedou ${ }^{\mathrm{a}}$, Kepa Zulueta Uriondo ${ }^{\mathrm{b}}$, Chi Nghia Chung ${ }^{\mathrm{c}}$, Hussein Rappel ${ }^{\mathrm{d}}$, Lars \\ Beex $^{\mathrm{d}}$, Laurent Adam ${ }^{\mathrm{e}}$, Aitor Arriaga ${ }^{\mathrm{b}}$, Zoltan Major ${ }^{\mathrm{c}}$, Ling $\mathrm{Wu}^{\mathrm{a}}$, Ludovic Noels ${ }^{\mathrm{a}, *}$ \\ ${ }^{a}$ University of Liege, Department of Mechanical and Aerospace Engineering, Computational $\mathcal{G}$ Multiscale Mechanics \\ of Materials, Allée de la découverte 9, B-4000 Liège, Belgium \\ ${ }^{b}$ Leartiker Polymer R\&BD, Xemein Etorbidea 12A, 48270 Markina-Xemein, Bizkaia, Spain \\ ${ }^{c}$ Johannes Kepler University Linz, Institute of Polymer Product Engineering, Altenbergerstrasse 69, 4040 Linz, \\ Austria \\ ${ }^{d}$ University of Luxembourg, Computational Mechanics Research Unit of Engineering Sciences, Maison du Nombre \\ 6, Avenue de la Fonte, 4364 Esch-sur-Alzette, Luxembourg \\ ${ }^{e}$ e-Xstream Engineering, Axis Park-Building H, Rue Emile Francqui 9, B-1435 Mont-Saint-Guibert, Belgium
}

\begin{abstract}
We present a stochastic approach combining Bayesian Inference (BI) with homogenization theories in order to identify, on the one hand, the parameters inherent to the model assumptions and, on the other hand, the composite material constituents behaviors, including their variability. In particular, we characterize the model parameters of a Mean-Field Homogenization (MFH) model and the elastic matrix behavior, including the inherent dispersion in its Young's modulus, of non-aligned Short Fibers Reinforced Polymer (SFRP) composites. The inference is achieved by considering as observations experimental tests conducted at the SFRP composite coupons level. The inferred model and material law parameters can in turn be used in Mean-Field Homogenization (MFH)-based multi-scale simulations and can predict the confidence range of the composite material responses.
\end{abstract}

Keywords: Multiscale, Stochastic, Composites, Bayesian inference, Inverse identification method

\section{Introduction}

Short Fibers Reinforced Polymer (SFRP) composites can be produced at low cost using the injection molding process, making them an increasingly popular material in several engineering

\footnotetext{
${ }^{*}$ Corresponding author

Email addresses: mohamedyahya72@gmail.com (Mohamed Mohamedou), kzulueta@leartiker.com (Kepa Zulueta Uriondo), Chi_Nghia.Chung@jku.at (Chi Nghia Chung), hussein.rappel@uni.lu (Hussein Rappel), lars.beex@uni.lu (Lars Beex), laurent.adam@e-xstream.com (Laurent Adam), aarriaga@leartiker.com (Aitor Arriaga), Zoltan.Major@jku.at (Zoltan Major), L.Wu@ulg.ac.be (Ling Wu ), L.Noels@ulg.ac.be (Ludovic Noels ) 
applications. However, the numerical simulation of composite structural applications remains challenging because of the heterogeneous nature of the material itself. In order to be predictive, structural simulations should be informed from the scale corresponding to the material constituents: the micro-structure geometrical parameters such as the inclusions aspect ratio, orientation and spatial distributions, but also the micro-constituents material responses.

In order to consider the micro-structure response and evolution during structural studies, multiscale methods, and in particular homogenization methods, are now commonly used, see the reviews in [1-3]. Among the different existing homogenization methods, Mean-Field Homogenization $(\mathrm{MFH})$ is a computationally efficient semi-analytical method for the modeling of multi-phase composites. MFH methods extend the Eshelby single inclusion solution [4] to multiple-inclusion interactions, such as in the Mori-Tanaka (M-T) scheme [5, 6] and in the self-consistent scheme [7, 8]. The methods were first derived for linear responses, but they can be extended to the non-linear range by defining a Linear Comparison Composite (LCC) [9-11] as a virtual heterogeneous material whose constituents linear behaviors correspond to the linearized behaviors of the real composite material constituents at a given strain state. MFH has also been developed in the context of SFRP by accounting for the misalignment of the fibers through their Orientation Distribution Function (ODF). In that context, pseudo-grains of aligned inclusions are first defined and homogenized using a M-T approach, and the different grain responses are then homogenized in a second step through a Voigt, i.e. uniform strain, assumption weighted using the ODF [12-14].

Although MFH has been shown to predict accurate results in comparison with full-field simulations at a much lower computation cost, for practical application the model parameters, fibers aspect ratio distribution, volume fraction, ODF, fiber material response and matrix material response, have to be identified first. This need, which is also a requirement for any other multiscale method, is complexified by the fact that the local properties vary from one location to the other in a component obtained by injection molding. Fibers ODF, or again volume fraction can be experimentally measured $[15,16]$ at different Locations, but the process is time consuming and can only be achieved once the component is manufactured and not at the design stage. As an alternative, the fiber ODF and volume fraction can be predicted through the process numerical simulation $[16,17]$. However, the use of ODF in 2-step homogenization usually relies on the assumption that the inclusions have a unique aspect ratio, which is not realistic. In that context an "effective" 
aspect ratio has to be defined by the user. Another difficulty is that material properties of the constituents are not always predictable and usually require complex experimental characterization, in particular in the non-linear regime. This is particularly true for a polymeric-based matrix phase since its material response strongly depends on the manufacturing process conditions. Besides, even for given environmental conditions, the material properties can vary by several percents. In [18], the tensile modulus of polyamide 6 (PA06) measured at ambient temperature and at constant loading rate of $5 \mathrm{~mm} \cdot \mathrm{min}^{-1}$ ranges from 1200 to $3400 \mathrm{MPa}$. For these reasons, the material behavior of the matrix phase is generally obtained using an inverse identification process from coupons experimental tests. However experimental measurements are entailed by uncertainties, either because of the measurement devices themselves that introduce errors, or because of existing natural deviations in the properties that are to be identified, which is the case for SFRP coupons. As an example, in [18], tensile tests conducted on 30\% of weight Glass fiber (GF) reinforced PA06 (PA06-GF30) lead to a Young's modulus ranging from 6200 to $9500 \mathrm{MPa}$.

There is thus a need to couple multiscale methods with statistical identification methods. When considering classical regression analyzes such as the Least Square Method (LSM), the confidence intervals are usually estimated under a normal distribution assumption [19], and part of the experimental test information is lost. On the contrary, Bayesian Inference (BI) [20] constitutes another framework in which uncertainties in identified parameters naturally arise from the identification process itself under the form of a Probability Density Function (PDF). Bayesian inference is structured around Bayes' theorem, in which the sought so-called posterior distribution function of the parameters to be identified is obtained from a prior distribution of the latter, which reflects the initial believe or knowledge one has, that is corrected using a likelihood function constructed from the different observation data, e.g. experimental results.

Starting from the work of [21], many works identified the parameters of material models through BI: elasticity constants of glass-fiber reinforced epoxy [22] and of carbon-epoxy unidirectional laminates [23] were inferred through vibration tests, and elasticity constants of graphite-epoxy laminates were identified from the displacement field in [24] through static tests; in the non-linear range, elasto-perfectly plastic model and cohesive zone parameters were inferred in [25], elasto-plastic material model parameters in [26, 27], visco-elasticity constants in $[28,29]$ and a hyperelastic model and its parameters in [30]; spatially varying, under the form of embedded inclusions, elasticity 
constants were identified in [31]; the list being non-exhaustive.

In the cited references, the identified parameters are related to either homogeneous or homogenized material models. Besides, the likelihood function is constructed by considering an error function [23], usually a Gaussian noise [24-26, 28-31], which could be estimated from the experimental device calibration, see the discussion in [26]. In that case, the underlying system is assumed to be deterministic and BI is seen as an identification mean accounting for the experimental noise.

BI was also used to account for the insufficient available information when constructing a stochastic model, such as polynomial-chaos-based stochastic methods [32, 33]. In this context, the coefficients of the Polynomial Chaos Expansion (PCE) are themselves random variables inferred using BI. Contrarily to the previously cited works, the system is considered explicitly as stochastic, and BI is used to infer the variables describing this stochasticity. With a similar view of characterizing a system stochasticity, material model parameters were considered as random variables following a Beta distribution in [34], and the distribution parameters were evaluated from observations using BI.

In this work, we intend to use BI to identify, from experimental tests conducted at the coupon level, the parameters of a micromechanics model that are either model related, resulting from model assumptions and that cannot be measured because of their abstract definition, or physically-based but that cannot be easily measured and can exhibit some variability. Among the first category, although the ODF is experimentally measured, we need to infer the inclusion aspect ratio that can be used in the model to predict results in good agreement with experimental observations. Among the second category, we need to infer the matrix elasticity constants of a MFH model, in which case direct measurements are not possible because the material is not at disposal. For the latter values, their variability should also be captured during the identification process in order for the multiscale model to be able to predict confidence ranges. The material system considered is a PA06 reinforced by short E-glass fibers (GF). Tests are conducted on $40 \%$ of weight GF reinforced PA06 (PA06GF40) coupons extracted at different Locations and with different Directions from several plates obtained by injection molding. Two approaches are successively considered for the BI. The first one uses a Gaussian noise to define the likelihood function, assuming that the observed discrepancies in the coupon tests result from experimental measurement errors. The second approach assumes that the micro-structure is characterized by uncertainties and that the dominant one is the matrix 
phase elasticity constant. We then represent the latter by a Beta distribution whose parameters are inferred from the experimental measurements. In order to assess the methodology, validation tests are conducted on coupons that were not used during the BI. Using the MFH model with the previously inferred parameters, we predict the confidence ranges corresponding to these validation experiments. It is shown that the first BI approach based on a Gaussian noise is able to evaluate an effective model-related parameter, i.e. the fibers aspect ratio, but not the variability observed in the physical parameters, i.e. the matrix properties, and as a result cannot predict a confidence range embedding the validation points, contrarily to the stochastic MFH obtained with the second BI approach.

The organization of the paper is as follows. The two-step MFH model is described in Section 2. Section 3 details the manufacturing process, the microstructural analysis, and the tensile tests of the composite coupons. It is then shown in Section 4 that to explain the discrepancies observed at the coupons level, a deterministic Young's modulus of the matrix phase cannot be considered. The two BI approaches to evaluate the effective fiber aspect ratio and the matrix Young's modulus and its uncertainties are successively developed in Section 5, and then applied on the experimental observations in Section 6, before drawing some conclusions in Section 7.

\section{Mean-field homogenization for non-aligned fiber-reinforced composites}

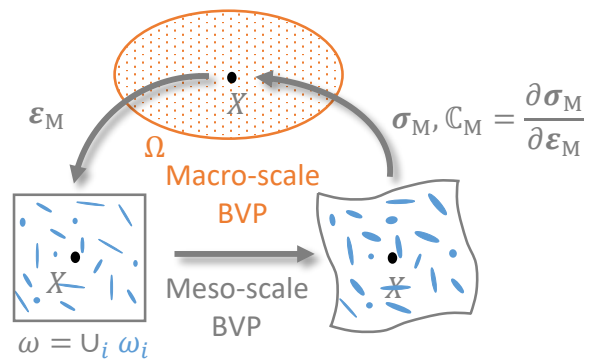

Figure 1: Homogenization-based multiscale method.

In a homogenization-based multiscale approach, the macro-scale structure $\Omega$ defines a boundaryvalue-problem (BVP) which is solved by considering homogenized material properties extracted, at each (macro) material point $\boldsymbol{X} \in \Omega$ of interest, from the resolution of a micro-scale BVP, see Fig. 1. This micro-scale BVP is defined on a micro-scale volume $\omega$ which represents the different 
phases $\omega_{i}$ of the material.

In order to define the micro-scale BVP, the relation between macro-strains $\varepsilon_{\mathrm{M}}$ and stresses $\boldsymbol{\sigma}_{\mathrm{M}}$ is transformed into a relation between the averaged values of the local strain tensor $\varepsilon_{\mathrm{m}}$ and of the local stress tensor $\boldsymbol{\sigma}_{\mathrm{m}}$ on $\omega$, with

$$
\varepsilon_{\mathrm{M}}=\left\langle\varepsilon_{\mathrm{m}}\right\rangle_{\omega} \quad \text { and } \quad \boldsymbol{\sigma}_{\mathrm{M}}=\left\langle\boldsymbol{\sigma}_{\mathrm{m}}\right\rangle_{\omega}
$$

where $\langle f(\boldsymbol{x})\rangle_{\omega}=\frac{1}{V_{\omega}} \int_{\omega} f(\boldsymbol{x}) \mathrm{d} V$, with $V_{\omega}$ the volume of $\omega$. The homogenized material tensor $\mathbb{C}_{\mathrm{M}}=\frac{\partial \sigma_{\mathrm{M}}}{\partial \varepsilon_{\mathrm{M}}}$ also results from the micro-scale BVP resolution.

In this work we rely upon MFH to conduct multiscale analyzes of SFRP composites and present first the general equations for two-phase linear composites with aligned uniform inclusions, before summarizing the two-step homogenization method for non-aligned inclusions.

\subsection{Mean-Field Homogenization (MFH) for two-phase linear composites}

Considering a two-phase composite material with the respective volume fractions $v_{0}+v_{\mathrm{I}}=1$, where the subscript 0 refers to the matrix and the subscript I to the aligned inclusions, the volume average over the micro-scale volume $\omega$ can be explicitly expressed in terms of the volume averages over the two phases $\omega_{0}$ and $\omega_{\mathrm{I}}$. Equations (1) thus become

$$
\varepsilon_{\mathrm{M}}=v_{0} \varepsilon_{0}+v_{\mathrm{I}} \varepsilon_{\mathrm{I}} \quad \text { and } \quad \boldsymbol{\sigma}_{\mathrm{M}}=v_{0} \boldsymbol{\sigma}_{0}+v_{\mathrm{I}} \boldsymbol{\sigma}_{\mathrm{I}}
$$

where we have used $\bullet_{i}$ to represent the volume average over the phase $\omega_{i}$, i.e. $\left\langle\bullet_{\mathrm{m}}\right\rangle_{\omega_{i}}$, for conciseness.

In the linear range, the phases responses are defined through the elastic stiffness tensors $\mathbb{C}_{i}^{\mathrm{el}}$ in phase $\omega_{i}$. The system of Eqs. (2) is then completed by assuming a relationship between the average responses of the different phases using a strain concentration tensor $\mathbb{B}^{\epsilon}$, i.e.

$$
\varepsilon_{\mathrm{I}}=\mathbb{B}^{\epsilon}\left(\mathrm{I}, \mathbb{C}_{0}^{\mathrm{el}}, \mathbb{C}_{\mathrm{I}}^{\mathrm{el}}\right): \varepsilon_{0}
$$

where "I" represents the geometry of the inclusions.

Using the linear elastic constitutive model in the phases, the set of Eqs. (2) and (3) is rewritten in a general constitutive expression for linear elastic composites as

$$
\boldsymbol{\sigma}_{\mathrm{M}}=\mathbb{C}_{\mathrm{M}}\left(\mathrm{I}, \mathbb{C}_{0}^{\mathrm{el}}, \mathbb{C}_{\mathrm{I}}^{\mathrm{el}}, v_{\mathrm{I}}\right): \varepsilon_{\mathrm{M}},
$$

with

$$
\mathbb{C}_{\mathrm{M}}=\left[v_{\mathrm{I}} \mathbb{C}_{\mathrm{I}}^{\mathrm{el}}: \mathbb{B}^{\epsilon}\left(\mathrm{I}, \mathbb{C}_{0}^{\mathrm{el}}, \mathbb{C}_{\mathrm{I}}^{\mathrm{el}}\right)+v_{0} \mathbb{C}_{0}^{\mathrm{el}}\right]:\left[v_{\mathrm{I}} \mathbb{B}^{\epsilon}\left(\mathrm{I}, \mathbb{C}_{0}^{\mathrm{el}}, \mathbb{C}_{\mathrm{I}}^{\mathrm{el}}\right)+v_{0} \mathbb{I}\right]^{-1}
$$




\subsection{Strain concentration tensor}

Different assumptions can be considered to define the strain concentration tensor $\mathbb{B}^{\epsilon}\left(\mathrm{I}, \mathbb{C}_{0}^{\mathrm{el}}, \mathbb{C}_{\mathrm{I}}^{\mathrm{el}}\right)$. In this work we will consider the following two

- The Mori-Tanaka [5] method (M-T) extends the single inclusion solution of Eshelby [4] to multiple-inclusion interacting by assuming that the average strain in the matrix phase corresponds to the strain at infinity of the single inclusion solution problem, i.e. for a two-phase composite material

$$
\mathbb{B}^{\epsilon}\left(\mathrm{I}, \mathbb{C}_{0}^{\mathrm{el}}, \mathbb{C}_{\mathrm{I}}^{\mathrm{el}}\right)=\left\{\mathbb{I}+\mathbb{S}:\left[\left(\mathbb{C}_{0}^{\mathrm{el}}\right)^{-1}: \mathbb{C}_{\mathrm{I}}^{\mathrm{el}}-\mathbb{I}\right]\right\}^{-1},
$$

where the Eshelby tensor [4] $\mathbb{S}\left(\mathrm{I}, \mathbb{C}_{0}^{\mathrm{el}}\right)$ depends on the geometry of the inclusion "I" and on the elastic tensor of the matrix phase $\mathbb{C}_{0}^{\mathrm{el}}$.

- The Voigt model assumes the same average strain in the different phases, i.e.

$$
\mathbb{B}^{\epsilon}=\mathbb{I}
$$

where $(\mathbb{I})_{i j k l}=\frac{1}{2}\left(\delta_{i k} \delta_{j l}+\delta_{i l} \delta_{j k}\right)$ is the identity fourth-order tensor.

\subsection{MFH for multi-phase composite materials}

\subsubsection{Orientation Distribution Function (ODF)}

For short-fiber reinforced composites produced by the injection molding process, although all the fibers are made of the same material, the composite material cannot be considered as being two-phase because of their misalignment and of the variation in their aspect ratio. In this work, each fiber of diameters $d$ is considered to be straight of length $l$ and characterized by an aspect ratio $a_{r}=\frac{l}{d}$, while its orientation is characterized by a unit vector $\boldsymbol{p}$ along the fiber axis.

For a collection of fibers, the complete description of orientations is obtained through a probability density function $\pi_{\boldsymbol{P}}(\boldsymbol{p})$, also called Orientation Distribution Function (ODF), such that $\pi_{\boldsymbol{P}}(\boldsymbol{p}) \mathrm{d} \boldsymbol{p}$ is the probability of a fiber to be oriented between $\boldsymbol{p}$ and $\boldsymbol{p}+\mathrm{d} \boldsymbol{p}$ and we have $\oint \pi_{\boldsymbol{P}}(\boldsymbol{p}) \mathrm{d} \boldsymbol{p}=1$. It is equivalent to the original expression written within the spherical coordinates

$$
\int_{\theta=0}^{\pi} \oint_{\phi=0}^{2 \pi} \pi_{\boldsymbol{P}}(\boldsymbol{p}(\theta, \phi)) \sin (\theta) \mathrm{d} \theta \mathrm{d} \phi=1
$$

where $\theta$ is the polar angle and $\phi$ is the azimuthal angle. In practice, the $\operatorname{ODF} \pi_{\boldsymbol{P}}(\boldsymbol{p})$ is not always directly available, and it is more common to have access to the ODF-weighted average of 
$\boldsymbol{p} \otimes \boldsymbol{p}$, where the symbol $\otimes$ designates a tensor or dyadic product, which is called the second-order orientation tensor

$$
\boldsymbol{a}=\oint \boldsymbol{p} \otimes \boldsymbol{p} \pi_{\boldsymbol{P}}(\boldsymbol{p}) \mathrm{d} \boldsymbol{p}
$$

Although homogenization methods can be constructed around the knowledge of the orientation tensor, see $[13,14,35]$, since the ODF does not differentiate fibers of different aspect ratio values, this requires the identification of an effective aspect ratio. Let us note that if the aspect ratio distributions are different along the different directions $\boldsymbol{p}$ such an effective aspect ratio value is not unique. In this work, we justify in Section 3 the use of a unique effective aspect ratio, which will be inferred from the experimental measurements in Section 5.

However, in the context of homogenization, the volume fraction of the fibers of aspect ratio $a_{r}$ along a direction $\boldsymbol{p}$ is more representative than the fibers count. We thus consider the variables $v_{f}, a_{r}, \boldsymbol{p}$ as the volume of one fiber $v_{f}$, its aspect ratio $a_{r}$ and its direction $\boldsymbol{p}$ whose probability function $\pi_{V_{f}, A_{r}, \boldsymbol{P}}$ will be evaluated in the next Section. We can then define the volume fraction $v^{\left(a_{r}, \boldsymbol{p}\right)} \pi_{A_{r}, \boldsymbol{P}}\left(a_{r}, \boldsymbol{p}\right)$ of fibers having an aspect ratio between $a_{r}$ and $a_{r}+\mathrm{d} a_{r}$ and oriented along a direction between $\boldsymbol{p}$ and $\boldsymbol{p}+\mathrm{d} \boldsymbol{p}$ with

$$
v^{\left(a_{r}, \boldsymbol{p}\right)}=\frac{\int_{\mathbb{R}^{+}} v_{f} \pi_{V_{f} \mid A_{r}, \boldsymbol{P}}\left(v_{f} \mid a_{r}, \boldsymbol{p}\right) \mathrm{d} v_{f}}{\iint_{\mathbb{R}^{+}} \int_{\mathbb{R}^{+}} v_{f} \pi_{V_{f}, A_{r}, \boldsymbol{P}}\left(v_{f}, a_{r}, \boldsymbol{p}\right) \mathrm{d} v_{f} \mathrm{~d} a_{r} \mathrm{~d} \boldsymbol{p}} .
$$

and with $\iint_{\mathbb{R}^{+}} v^{\left(a_{r}, \boldsymbol{p}\right)} \pi_{A_{r}, \boldsymbol{P}}\left(a_{r}, \boldsymbol{p}\right) \mathrm{d} a_{r} \mathrm{~d} \boldsymbol{p}=1$.

\subsubsection{Two-step homogenization}

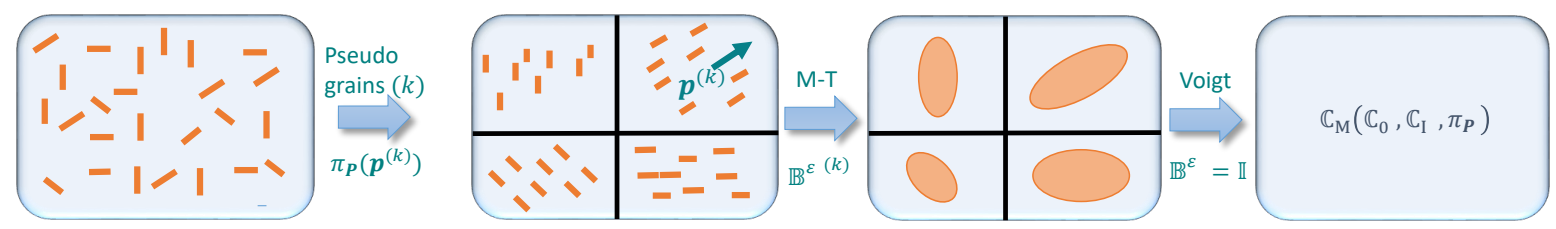

Figure 2: Two-step homogenization: the micro-scale volume element $\omega$ is first decomposed into a set of pseudo-grains $\omega^{(k)}$ homogenized using the M-T assumption; the homogenized composite material behavior is then obtained using the Voigt assumption.

When considering such a material with inclusions having different orientations or shapes, one could envisioned a direct (single step) homogenization based on an extension of Mori-Tanaka (MT). However, Benveniste et al. [36] proved that in linear elasticity the macro stiffness tensor has 
the required symmetries only if all the inclusions are aligned and similarly shaped. Otherwise, a direct M-T homogenization should be avoided as it might lead to physically unacceptable results. Accurate and physically acceptable results are thus provided by a two-step homogenization strategy [12-14], as illustrated in Fig. 2. In this work we consider a two-step homogenization accounting for the ODF, and which is based on:

- The decomposition in phases (pseudo-grains) $\omega^{(k)}$ where the inclusions "I ${ }^{(k)}$ " have the same aspect ratio $a_{r}$, and the same orientation defined by the direction $\boldsymbol{p}^{(k)}$. Each phase is then defined on the assumption that the inclusions see the same volume fraction of matrix $v_{0}$;

- The homogenization on the aggregate of pseudo-grain $\omega^{(k)}$, with the set of Eq. (2-3) rewritten as

$$
\begin{aligned}
\langle\varepsilon\rangle_{\omega^{(k)}} & =v_{0}\left\langle\varepsilon_{0}\right\rangle_{\omega^{(k)}}+v_{\mathrm{I}}\left\langle\varepsilon_{\mathrm{I}}\right\rangle_{\omega^{(k)}}, \\
\langle\boldsymbol{\sigma}\rangle_{\omega^{(k)}} & =v_{0}\left\langle\boldsymbol{\sigma}_{0}\right\rangle_{\omega^{(k)}}+v_{\mathrm{I}}\left\langle\boldsymbol{\sigma}_{\mathrm{I}}\right\rangle_{\omega^{(k)}}, \\
\left\langle\varepsilon_{\mathrm{I}}\right\rangle_{\omega^{(k)}} & =\mathbb{B}^{\epsilon}\left(\mathrm{I}^{(k)}, \mathbb{C}_{0}^{\mathrm{el}}, \mathbb{C}_{\mathrm{I}}^{\mathrm{el}}\right):\left\langle\varepsilon_{0}\right\rangle_{\omega^{(k)}},
\end{aligned}
$$

and the linear operator (5) rewritten as

$$
\mathbb{C}^{(k)}=\left[v_{\mathrm{I}} \mathbb{C}_{\mathrm{I}}^{\mathrm{el}}: \mathbb{B}^{\epsilon}\left(\mathrm{I}^{(k)}, \mathbb{C}_{0}^{\mathrm{el}}, \mathbb{C}_{\mathrm{I}}^{\mathrm{el}}\right)+v_{0} \mathbb{C}_{0}^{\mathrm{el}}\right]:\left[v_{\mathrm{I}} \mathbb{B}^{\epsilon}\left(\mathrm{I}^{(k)}, \mathbb{C}_{0}^{\mathrm{el}}, \mathbb{C}_{\mathrm{I}}^{\mathrm{el}}\right)+v_{0} \mathbb{I}\right]^{-1},
$$

completed by the M-T strain concentration tensor (6);

- The homogenization of all phase (pseudo-grain) $\omega^{(k)}$ using Voigt strain concentration tensor (7), in which case the set of Eq. (2-3) is rewritten as

$$
\langle\varepsilon\rangle_{\omega}(k)=\langle\varepsilon\rangle_{\omega}=\varepsilon_{\mathrm{M}} \quad \forall \omega^{(k)},
$$

with the homogenized stress evaluated by

$$
\boldsymbol{\sigma}_{\mathrm{M}}=\langle\boldsymbol{\sigma}\rangle_{\omega}=\oint\langle\boldsymbol{\sigma}\rangle_{\omega^{(k)}} \pi_{\boldsymbol{P}}\left(\boldsymbol{p}^{(k)}\right) \mathrm{d} \boldsymbol{p}^{(k)} \simeq \sum_{k}\langle\boldsymbol{\sigma}\rangle_{\omega^{(k)}} \pi_{\boldsymbol{P}}\left(\boldsymbol{p}^{(k)}\right) \Delta \boldsymbol{p}^{(k)}
$$

and the linear operator (5) by

$$
\mathbb{C}_{\mathrm{M}}=\oint \frac{\partial\langle\boldsymbol{\sigma}\rangle_{\omega^{(k)}}}{\partial \varepsilon_{\mathrm{M}}} \pi_{\boldsymbol{P}}\left(\boldsymbol{p}^{(k)}\right) \mathrm{d} \boldsymbol{p}^{(k)} \simeq \sum_{k} \mathbb{C}^{(k)} \pi_{\boldsymbol{P}}\left(\boldsymbol{p}^{(k)}\right) \Delta \boldsymbol{p}^{(k)}
$$




\subsubsection{Mathematical description of the fiber orientation}

In practice, the ODF $\pi_{\boldsymbol{P}}(\boldsymbol{p})$ is not directly available for short fiber reinforced composites obtained using injection molding. Besides the second-order orientation tensor (9), in order to evaluate the ODF function, one needs the ODF-weighted average of $\boldsymbol{p} \otimes \boldsymbol{p} \otimes \boldsymbol{p} \otimes \boldsymbol{p}$, which is called the fourthorder orientation tensor

$$
\mathbb{A}=\oint \boldsymbol{p} \otimes \boldsymbol{p} \otimes \boldsymbol{p} \otimes \boldsymbol{p} \pi_{\boldsymbol{P}}(\boldsymbol{p}) \mathrm{d} \boldsymbol{p} .
$$

However, the fourth-order orientation tensor $\mathbb{A}$ can only be deduced exactly from the second orientation tensor $\boldsymbol{a}$ in the case of aligned or randomly oriented fibers. In this work, we consider the interpolation method developed in $[13,14]$ and reported in Appendix A.

Once the second- and fourth-order tensors $\boldsymbol{a}$ and $\mathbb{A}$ have been evaluated, the ODF $\pi_{\boldsymbol{P}}(\boldsymbol{p})$ can be reconstructed using the method developed by Onat and Leckie [37] and summarized in Appendix B. The ODF may be used to average either the stress tensor following Eq. (16) or the linear operator following Eq. (18). Since in practice these integrals are computed numerically, a discrete form of the ODF should be provided, in particular to evaluate $\Delta \boldsymbol{p}^{(k)}$. To this end, we have implemented the algorithm that has been proposed by Weber et al. in [35], see Appendix C. In this approach, the unit orientation vector $\boldsymbol{p}\left(\theta^{(i)}, \phi^{(j)}\right)$ is viewed as the outer unit normal to a facet $(k)$, of surface $S\left(\theta^{(i)}, \phi^{(j)}\right)$ of the unit sphere described in the spherical coordinate system of polar angle $\theta$ and azimuthal angle $\phi$. In particular, the linear operator (18) is then evaluated as

$$
\mathbb{C}_{\mathrm{M}} \approx 2 \sum_{i}^{N_{\theta}} \sum_{j}^{N_{\phi}^{(i)}} \mathbb{C}\left(\boldsymbol{p}\left(\theta^{(i)}, \phi^{(j)}\right)\right) \pi_{\boldsymbol{P}}\left(\boldsymbol{p}\left(\theta^{(i)}, \phi^{(j)}\right)\right) S\left(\theta_{i}, \phi_{j}\right)
$$

where $N_{\theta}$ and $N_{\phi}^{(i)}$ designate the total numbers of subdivisions along the $\theta$ and $\phi(\theta)$ angles, respectively.

\section{Experimental tests}

Table 1: PA06-GF constituents material properties. In [18] PA06 was tested at ambient temperature and a strain rate of $5 \mathrm{~mm} \cdot \mathrm{min}^{-1}$. PA06 manufacturer value [38] at $23^{\circ}$ is also reported.

\begin{tabular}{l|ccc}
\hline Phase & Density $\left[\mathrm{Kg} \cdot \mathrm{m}^{3}\right]$ & Young's modulus $[\mathrm{MPa}]$ & Poisson coefficient [-] \\
\hline E-glass fiber [18] & 2600 & 72000 to 73000 & 0.22 \\
PA06 & $1130[18]$ & 1200 to $3400[18] / 3600[38]$ & $0.39[39]$ \\
\hline
\end{tabular}


The material system considered in this work is a polyamide 6 (PA06) matrix reinforced by short E-glass fibers (GF), with $40 \%$ of weight GF (PA06-GF40).

Concerning the GF, a good estimation of their mechanical properties can be found in the literature and the values evaluated in [18] are reported in Table 1. Concerning the PA06 material, on the one hand the in-situ properties of the composite material are not easily determined, and on the other hand experimental tests show a large range as reported in [18], see Table 1. Besides, manufacturer provided value [38] is above this observed range. For these two reasons, the purpose of this paper is to infer them, including their variation range, from coupon tests. In this section, we describe the manufacturing process of the coupons, their characterization in terms of microstructures, and finally the tensile tests that have been conducted.

\subsection{Coupons manufacturing process}

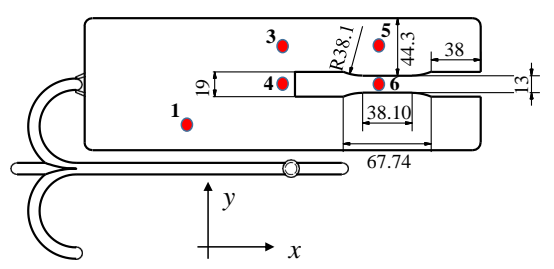

(a) $0^{\circ}$-coupon

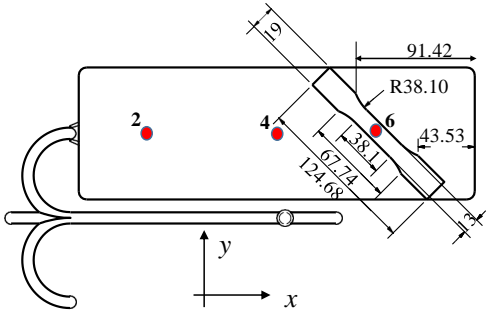

(b) $45^{\circ}$-coupon

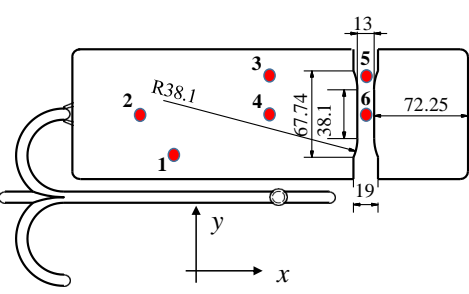

(c) $90^{\circ}$-coupon

Figure 3: Locations and geometries (in $\mathrm{mm}$ ) of the tested coupons along (a) $0^{\circ}$-traction; (b) $45^{\circ}$-traction; and (c) $90^{\circ}$-traction

The injection molding of composite $304.8 \times 101.6 \times 3.65 \pm 0.02 \mathrm{~mm}^{3}$-plates has been performed in an Engel Insert v200 Single machine. The 200-ton clamping force and $55 \mathrm{~mm}$ diameter screw allowed the application of 110-bar injection pressure and 50-bar packing pressure on the plates. Being the matrix a PA6, the nozzle temperature has been set at $270^{\circ} \mathrm{C}$ and the mold's temperature at $90^{\circ} \mathrm{C}$. The plates have been injected in 2 seconds and the packing pressure has been applied during 50 seconds.

Coupons were then cut from the plates along 3 different Directions, $0^{\circ}, 45^{\circ}$ and $90^{\circ}$, and centered at 6 different Locations as illustrated in Fig. 3. 
Table 2: Orientation tensor $\boldsymbol{a}$ at each Location for the PA06-GF40 material system. The standard deviation is reported for Locations measured on several plates.

\begin{tabular}{l|cccccc}
\hline Location & $\# 1$ & $\# 2$ & $\# 3$ & $\# 4$ & $\# 5$ & $\# 6$ \\
\hline$a_{11}$ & 0.712 & $0.653 \pm 0.009$ & 0.778 & 0.747 & 0.747 & $0.714 \pm 0.017$ \\
$a_{22}$ & 0.255 & $0.312 \pm 0.008$ & 0.172 & 0.204 & 0.191 & $0.248 \pm 0.016$ \\
$a_{33}$ & 0.033 & $0.035 \pm 0.001$ & 0.051 & 0.049 & 0.052 & $0.038 \pm 0.002$ \\
$a_{12}$ & -0.015 & $-0.008 \pm 0.008$ & 0.004 & 0.0031 & -0.066 & $-0.052 \pm 0.007$ \\
$a_{13}$ & -0.001 & $-0.001 \pm 0.001$ & -0.005 & 0.005 & -0.003 & $0.001 \pm 0.001$ \\
$a_{23}$ & 0.005 & $-0.005 \pm 0.001$ & 0 & 0.003 & 0.002 & $-0.001 \pm 0.001$ \\
\hline
\end{tabular}

\subsection{Micro-structure characterization}

First the fiber orientation, at the 6 different Locations illustrated in Fig. 3 of the PA06-GF40 plates, has been characterized by computed tomography technique. The Computed Tomography $(\mathrm{CT})$ is a nondestructive test for analyzing the microstructure of samples. It is based on the difference in the density of the constituents of the material. An x-ray emitter attacks the sample under analysis and the generated shadow is analyzed afterwards. The different gray shadows show different material densities which allow the identification of the heterogeneity of the material. In this project, a GE phoenix Nanoton 180 NF machine has been employed in order to scan 2 cubicmicrons material samples. The measurements have been conducted on volume of $2 \times 2 \times 3.2 \mathrm{~mm}^{3}$ at each of the 6 Locations. For Locations \#2 and \#6 they have been repeated on three different plates. The second-order orientation tensors $\boldsymbol{a}$ deduced from the CT-scan samples are reported in Table 2. It can be seen that Location \#3 at border of the plate exhibits a better alignment (higher $\left.a_{11}\right)$ than Locations near the plate center.

Nevertheless, as explained in Section 2.3.1, applying the ODF in the two-step homogenization process assumes that the populations of fibers have the same aspect ratio $a_{r}$. When considering Location \#6 of Plate \#3, analyzing the conditional distributions $\pi_{A_{r} \mid \boldsymbol{P}}\left(a_{r} \mid \boldsymbol{p}\right)$ of fiber aspect ratio $a_{r}$ for different facet directions $\boldsymbol{p}$ in terms of the spherical polar angle $\theta$ and azimuthal angle $\phi$, see Figs. 4(a)-4(c), it appears that the fibers tend to have a higher aspect ratio when aligned in the plate plane $\left(\theta=\frac{\pi}{2}\right)$. Since most of the fibers are aligned in this plane, see Table 2 , and since there is no obvious variation with respect to the angle $\phi$, we can assume that the fiber aspect ratio distribution for $\left(\theta=\frac{\pi}{2}, \phi=0\right)$ is representative of the plate. 


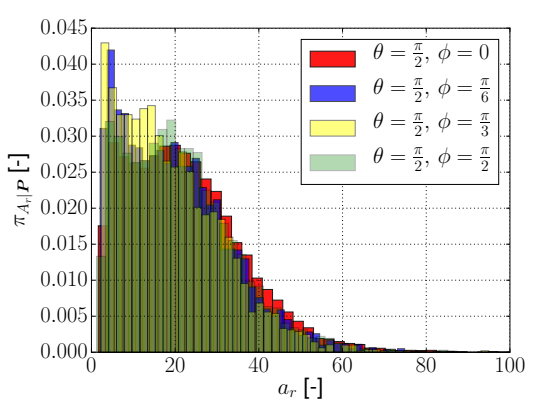

(a) $\pi_{A_{r} \mid \boldsymbol{P}}\left(a_{r} \mid \theta=\frac{\pi}{2}\right)$

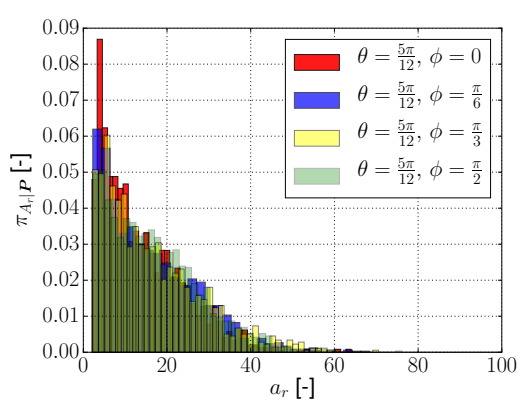

(b) $\pi_{A_{r} \mid \boldsymbol{P}}\left(a_{r} \mid \theta=\frac{5 \pi}{12}\right)$

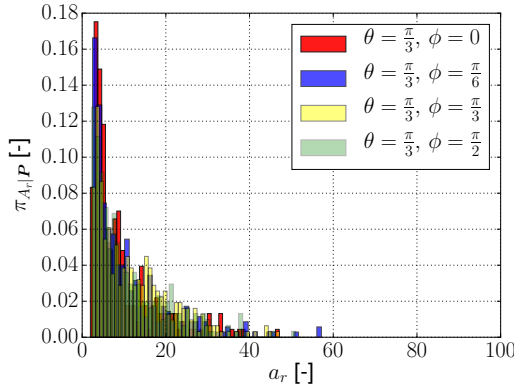

(c) $\pi_{A_{r} \mid \boldsymbol{P}}\left(a_{r} \mid \theta=\frac{\pi}{3}\right)$

Figure 4: Conditional distributions $\pi_{A_{r} \mid \boldsymbol{P}}$ of the fiber aspect ratio $a_{r}$ at Location \#6, Plate \#3, for different facet orientations in terms of spherical polar angle $\theta$ and azimuthal angle $\phi$

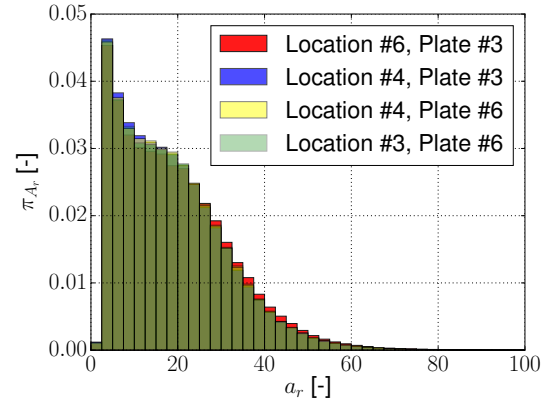

(a) $\pi_{A_{r}}\left(a_{r}\right)$

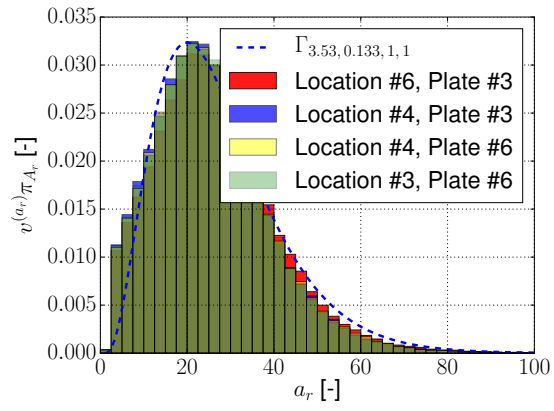

(b) $v^{\left(a_{r}\right)} \pi_{A_{r}}\left(a_{r}\right)$

Figure 5: Fiber aspect ratio distributions for different Plates and at different Plate Locations: (a) fiber aspect ratio distribution $\pi_{A_{r}}$ in terms of fibers count; and (b) distribution of volume fraction $v^{\left(a_{r}\right)} \pi_{A_{r}}$ of inclusions of aspect ratio $a_{r}$.

Since there exists a distribution of aspect ratio, it is not obvious which value should be used during the MFH process, see Section 2.1. The distribution $\pi_{A_{r} \mid \boldsymbol{P}}\left(a_{r} \mid \boldsymbol{p}\right)$ cannot directly be used in a two-step homogenization process since it is related to a fiber count, and not to a fiber volume fraction, in which case the effect of the small fibers would be overestimated. One could account for the difference of volume values for the different aspect ratio values in order to define the volume fraction of pseudo-grains of different aspect ratio values. However, we note that the definition of the ODF would not be fully compatible since it is also based on a fiber count. In that case it would be more meaningful to consider the distribution $v^{\left(a_{r}\right)} \pi_{A_{r}}=\int v^{\left(a_{r}, \boldsymbol{p}\right)} \pi_{A_{r}, \boldsymbol{P}}\left(a_{r}, \boldsymbol{p}\right) \mathrm{d} \boldsymbol{p}$ of the volume fraction of inclusions having an aspect ratio $a_{r}$, obtained from Eq. (10) and illustrated in Fig. 5(b). Besides, it is an overwhelming process, both during the model definition and resolution. Instead, in this paper we define an effective value $a_{r}$, which should be inferred from experimental data. We 
consider it as a unique value since, on the one hand, we have justified that the distribution for $\left(\theta=\frac{\pi}{2}, \phi=0\right)$ is representative of a given Plate and since, on the other hand, the aspect ratio distribution $\pi_{A_{r} \mid \boldsymbol{P}}\left(a_{r} \mid \boldsymbol{p}\right)$ is found to be insensitive to the Plate and Plate Location, see Fig. 5(a).

We also note that during injection molding there exists a skin core effect such that the orientation tensor is not uniform across the plate thickness. Since in this work we use a two-step MFH process, see Section 2.1, in which different orientation populations are clustered depending on their orientation before applying a Voigt assumption (identical strain), only the average orientation tensor across the plate thickness is required. Indeed, evaluating the different orientation tensors across the thickness and then performing several MFH would lead to the same results.

Table 3: Measured fiber mass fraction $m_{\mathrm{I}}$ and deduced fiber volume fraction $v_{\mathrm{I}}$ at each Location for the PA06-GF40 material system. Their standard deviations are also reported.

\begin{tabular}{l|cccccc}
\hline Location & $\# 1$ & $\# 2$ & $\# 3$ & $\# 4$ & $\# 5$ & $\# 6$ \\
\hline$m_{\text {I }}(\%)$ & $39.85 \pm 0.07$ & $40.19 \pm 0.31$ & $39.26 \pm 0.05$ & $39.55 \pm 0.25$ & $39.21 \pm 0.09$ & $39.32 \pm 0.18$ \\
$v_{\text {I }}(\%)$ & $22.36 \pm 0.03$ & $22.60 \pm 0.14$ & $21.93 \pm 0.02$ & $22.14 \pm 0.11$ & $21.90 \pm 0.04$ & $21.97 \pm 0.08$ \\
\hline
\end{tabular}

The mass fraction $m_{\mathrm{I}}$ of fibers has been measured at each Location of the PA06-GF40 plates using the pyrolysis technique by considering three through the thickness samples from three different Plates. The volume fraction $v_{\mathrm{I}}$ is then deduced from the densities reported in Table 1 , and is reported in Table 3 .

\subsection{Tensile tests}

The tensile tests have been performed in a MTS Insight electromechanical actuator. This vertical machine is capable of applying up to $100 \mathrm{kN}$ force for performing quasi static tests. The strain measurements, were carried out by means of a MTS Clip On extensometer. The machine is equipped with mechanical grips for holding the specimens. In the case of a load cell of the testing machine, the relative error of the resolution is $0.01 \%$. In the case of the extensometer used for measuring the strain the error of resolution is below $0.009 \%$.

Tests were conducted at $23^{\circ} \mathrm{C}$ at a strain rate of $1 \mathrm{~mm} \cdot \mathrm{min}^{-1}$. For each Location and Direction couple illustrated in Fig. 3, three coupons of dimensions reported in Fig. 3 were extracted from different composite Plates and tested under uni-axial tension. The extracted composite material Young's modulii for the PA06-GF40 material system are reported in Table 4. We note that the 
Table 4: Measured composite Young's modulii $E_{c}(\mathrm{GPa})$ for different Plates, at different Locations and for different loading Directions.

\begin{tabular}{l|cccccc}
\hline Material & PA06-GF40 & & & & & \\
\hline Location & $\# 1$ & $\# 2$ & $\# 3$ & $\# 4$ & $\# 5$ & $\# 6$ \\
\hline $0^{\circ}$ & 12.089 & - & 11.663 & 11.370 & 11.658 & 11.292 \\
& 11.470 & - & 12.114 & 11.756 & 10.525 & 11.301 \\
& 13.526 & - & 12.032 & 11.891 & 10.441 & 10.772 \\
\hline \multirow{2}{*}{$45^{\circ}$} & - & 7.264 & - & 6.393 & - & 6.993 \\
& - & 7.043 & - & 6.291 & - & 7.776 \\
& - & 8.076 & - & 7.733 & - & 7.322 \\
\hline \multirow{2}{*}{$90^{\circ}$} & - & 7.339 & - & 6.020 & - & 6.348 \\
& - & 7.915 & - & 5.672 & - & 6.289 \\
& - & 7.803 & - & 5.750 & - & 6.073 \\
\hline
\end{tabular}

measurement errors due to the load cell and extensometer cannot explain the discrepancy observed in these values.

\section{Micro-mechanical parameters uncertainty impact}

In this section, we estimate the impact of the discrepancy observed on different measured micromechanical model parameters on the prediction of the composite Young's modulus using the MFH framework presented in Section 2, including the effective aspect ratio $a_{r}$ to be inferred. It is shown that the discrepancy observed in Table 4 can only result either from experimental measurement errors or from the existence of variability in the matrix Young's modulus.

We consider the composite Young's modulus observations $E_{c}^{(m)}$ reported in Table 4, where $(m)$ indexes all the $n_{\text {coupons }}$ coupons cut at different Locations $(i)$, with $i=1 . . n_{\text {pos }}$ and $n_{\text {pos }}=6$, and along different Directions $\psi^{(j)}$ with $j=1 . . n_{\text {dir }}^{(i)}$ and $n_{\text {dir }}^{(i)}$ up to three. Considering the microstructure parameters measured in Section 3.2 and assuming a value of the effective aspect ratio $a_{r}$, we can evaluate the corresponding matrix Young's modulii following the MFH model:

$$
E_{0 c}^{(m)}=\left(E_{\mathrm{MFH}}\right)^{-1}\left(E_{c}^{(m)} ; \psi^{(j)}, \mathrm{I}^{(i)}, v_{\mathrm{I}}^{(i)}\right) \quad \forall i \in\left[1 . . n_{\mathrm{pos}}\right] \text { and } j \in\left[1 . . n_{\mathrm{dir}_{i}}\right],
$$

where $E_{c}^{(m)}$ is the measured Young's modulus, and where "I ${ }^{(i)}$ " represents the inclusions in the different pseudo-grains at Location $i$. We note that the transformation $\left(E_{\mathrm{MFH}}\right)^{-1}$ always exists 


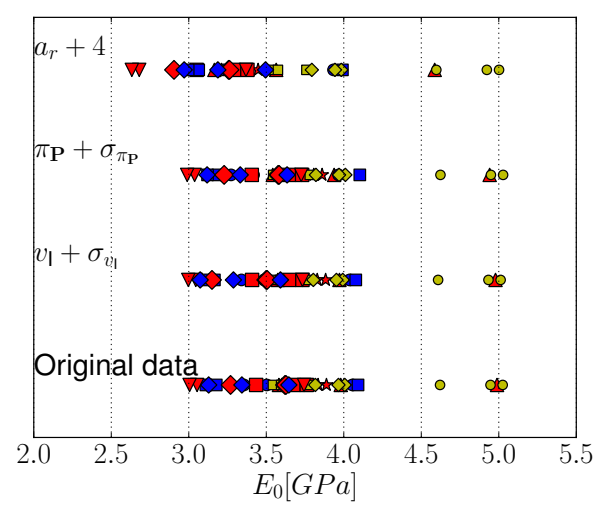

Figure 6: Effect of the micro-structural parameter on the matrix Young's modulus $E_{0}$ identification. "Original data" refers to the case of average microstructural parameters with an effective aspect ratio $a_{r}=16$; " $v_{\mathrm{I}}+\sigma_{\sigma}$ " refers to the case in which the volume fraction has been increased by its standard deviation; " $\boldsymbol{a}+\sigma_{\boldsymbol{a}}$ " refers to the case in which the orientation tensor has been modified by its standard deviation; $a_{r}+4$ refers to the case in which the effective aspect ratio has been modified. The symbol " $\triangle$ " refers to the values extracted at Location \#1, "०" at Location \#2, "*" at Location \#3, " $\square$ " at Location \#4, " $\nabla$ " at Location \#5, and " $\diamond "$ at Location \#6. The red color refers to loading along the $0^{\circ}$-Direction, blue along the $45^{\circ}$-Direction, and yellow along the $90^{\circ}$-Direction.

since $E_{\mathrm{MFH}}$ is monotonically increasing with $E_{0}$.

Considering the different Locations, the matrix Young's modulii $E_{0 c}^{(m)}$ are reported in Fig. 6 under the label "Original data". It can be seen that, for an aspect ratio $a_{r}=16$, the identified values range from about $2.8 \mathrm{GPa}$ to a bit more than $4 \mathrm{GPa}$, which is a range to be compared with the value provided by the manufacturer (3.6 GPa [38]) and which is of comparable width and order of magnitude as the one reported in Table 1 . The only values out of that range correspond to the loading along the $90^{\circ}$-Direction at Location \#2, and one value at Location $\# 1$ along the $0^{\circ}$-Direction. However these measurements are not reliable, see Table 4 , since for Location \#2, the composite Young's modulii along the $90^{\circ}$-Direction are larger than along the $45^{\circ}$-Direction, and since for Location \#1, one coupon Young's modulus is $10 \%$ higher. A more complete microcharacterization should be performed on these samples, and for these reasons, in the following we will not consider them. Besides, we note that considering only the $0^{\circ}$-Direction, the red symbols, spans a range from 2.8 $\mathrm{GPa}$ to $3.8 \mathrm{GPa}$. Similarly, considering the tests conducted at Location \#4 in all the Directions, the " $\square$ " symbols, spans a range from 3.1 GPa to almost 4.1 GPa. Such a range can thus only be explained by a variability in the matrix properties or in the multiscale model parameters. 
We then add successively to the volume fraction and to the ODF the measured standard deviations reported in Table 3 . We also increase the effective aspect ratio $a_{r}$ to 20 . The corresponding matrix Young's modulii $E_{0 c}^{(m)}$ are reported in Fig. 6, and it can be seen that the range of variation is not strongly affected by the uncertainties on the other micro-structural parameters, in comparison with the uncertainties on $E_{0 c}^{(m)}$. The main affecting parameter is the aspect ratio $a_{r}$, which tends to widen the range of Young's modulus when increased.

\section{Bayesian inference of the model parameters}

Following the analysis in Section 4, the discrepancy observed in Table 4 can only result either from experimental measurement error or from the existence of variability in the matrix Young's modulus. However, as detailed in Section 3.3, the measurement errors due to the load cell and extensometer remain lower than $0.01 \%$, motivating the identification of the matrix Young's modulus discrepancy. Nevertheless, the effective ratio $a_{r}$ to be used in the multiscale model should also be inferred.

Therefore, after having briefly recalled Bayes' theorem, the model parameter, i.e. the aspect ratio, and the physical parameter, i.e. the matrix Young's modulus, are inferred, first assuming the existence of a Gaussian noise as done in several references for parameters identification [2426, 28-31], and then by assuming the existence of stochasticity in the matrix Young's modulus.

\subsection{Bayesian inference (BI) theory}

Bayesian inference is a statistical analysis approach based on Bayes' theorem according to which the posterior probability of a random parameters vector $\boldsymbol{x} \in \mathbb{R}^{n}$ for given observations of another random vector $\boldsymbol{y} \in \mathbb{R}^{m}$ is proportional to the prior probability of the parameters vector $\boldsymbol{x}$ multiplied by the likelihood of $\boldsymbol{y}$ given observations of the random vector $\boldsymbol{x}$ :

$$
\pi(\boldsymbol{x} \mid \boldsymbol{y})=\frac{\pi(\boldsymbol{x}) \pi(\boldsymbol{y} \mid \boldsymbol{x})}{\pi(\boldsymbol{y})},
$$

where $\pi(\bullet)(\pi(\bullet \bullet \bullet))$ denotes a (conditional) Probability Density Function (PDF).

When applying Bayes' theory in the context of parameters identification, $\pi(\boldsymbol{x})$ is the prior distribution and reflects the initial believe or knowledge one has on $\boldsymbol{x}$. The conditional PDF $\pi(\boldsymbol{y} \mid \boldsymbol{x})$ is the law of observation or likelihood function, which is constructed from the different observation data. The conditional PDF $\pi(\boldsymbol{x} \mid \boldsymbol{y})$ is the posterior distribution of the random vector 
$\boldsymbol{x}$ that accounts for the observation data. Finally $\pi(\boldsymbol{y})$ is the PDF of the observation data. Since the latter is a constant for given observations, we simply write

$$
\pi_{\text {post }}(\boldsymbol{x} \mid \boldsymbol{y}) \propto \pi(\boldsymbol{y} \mid \boldsymbol{x}) \pi_{\text {prior }}(\boldsymbol{x})
$$

where we have added the subscripts "prior" and "post" to the corresponding distributions. In order to evaluate the posterior distribution (22), it is common to use MCMC techniques, which are analogous to a random walk in the parameter space $\boldsymbol{x} \in \mathbb{R}^{n}$. This work uses the adaptive variant [40] of the Metropolis algorithm [41], which is summarized in Appendix D.

Two cases are now considered to build the likelihood function.

\subsection{Error-based inference}

In the present context, the values to be inferred are the matrix Young's modulus $E_{0}$ and the effective aspect ratio $a_{r}$ to be used in the 2 -step MFH process, i.e. $\boldsymbol{x}=\left[E_{0}, a_{r}\right]$. The only a priori known information about the matrix Young's modulus is related to experimental measurements range and the average value provided by the manufacturer as reported in Table 1, and to the inverse identification conducted in Fig. 6. Since physically the value should remain positive and cannot be much larger than a few GPa we use a Gamma distribution as prior with

$$
\Gamma_{\alpha, \beta, a, c}(y)=\frac{\left(\frac{y-a}{c}\right)^{\alpha-1} \beta^{\alpha} e^{-\beta\left(\frac{y-a}{c}\right)}}{c \Gamma(\alpha)},
$$

where $\Gamma(\alpha)$ is the normalization constant, $a$ is the lower bound and $c$ allows defining the distribution independently of the variable units. We consider the Gamma distribution $\Gamma_{1.75}, 0.5,0.1 \mathrm{GPa}, 1 \mathrm{GPa}$ with its shape and rate parameters such that its expectation corresponds to the average value provided by the manufacturer. Considering the model aspect ratio, the a priori known information comes from the CT scan in terms of $v^{\left(a_{r}\right)} \pi_{A_{r}}$ since the distribution in terms of volume fraction is more meaningful than in terms of fiber counts. This distribution is illustrated in Fig. 5(b) in which a Gamma-distribution approximation is also displayed. A lower bound has been introduced in the latter distribution since the aspect ratio is defined such that $a_{r} \geq 1$. Eventually, the prior reads

$$
\pi_{\text {prior }}\left(\left[E_{0}, a_{r}\right]\right)=\Gamma_{1.75,0.5,0.1 \mathrm{GPa}, 1 \mathrm{GPa}} \times \Gamma_{3.53,0.133,1,1} .
$$

The random observation $y=\hat{E}_{c}$ gathers the experimental measurements $E_{c}^{(i, j, k)}$ reported in Table 4 , where $i=1 . . n_{\text {pos }}$ refers to the plate Location from which the coupons are cut, $j=1 . . n_{\text {dir }}^{(i)}$ 
refers to the Direction $\psi^{(j)}$ along which the coupon is cut at Location $(i)$, and where $k=1 . . n_{\text {sample }}^{(i, j)}$ refers to the number of samples cut along Direction $\psi^{(j)}$ at Location $(i)$.

Besides, the composite material Young's modulus along Direction $\psi^{(j)}$ at Location $(i)$ can be evaluated from the MFH model described in Section 2, following

$$
E_{\mathrm{MFH}}^{(i, j)}\left(\left[E_{0}, a_{r}\right]\right)=E_{\mathrm{MFH}}\left(E_{0}, \mathrm{I}^{(i)}\left(a_{r}\right) ; \psi^{(j)}, v_{\mathrm{I}}^{(i)}\right),
$$

with the inclusions $\mathrm{I}^{(i)}$ in the different pseudo-grains and the fiber volume fraction $v_{\mathrm{I}}^{(i)}$ evaluated at Location \# $(i)$. The composite material Young's modulus is then written as the sum of the MFH prediction and an error, or noise, $n^{(i, j)}$ which is related to the Location and loading Direction, with

$$
E_{c}^{(i, j)}=E_{\mathrm{MFH}}^{(i, j)}\left(\left[E_{0}, a_{r}\right]\right)+n^{(i, j)} .
$$

Since the different measurements on the coupons $(k)$ are independent, one can write the likelihood function for the tests along Direction $\psi^{(j)}$ at Location $(i)$ as

$$
\pi\left(\hat{E}_{c}^{(i, j)} \mid\left[E_{0}, a_{r}\right]\right)=\prod_{k=1}^{\substack{(i, j) \\ \text { sample }}} \pi_{n^{(i, j)}}\left[E_{c}^{(i, j, k)}-E_{\mathrm{MFH}}^{(i, j)}\left(\left[E_{0}, a_{r}\right]\right)\right]
$$

where $\hat{E}_{c}^{(i, j)}$ gathers the observation $E_{c}^{(i, j, k)}$ with $k=1 . . n_{\text {sample }}^{(i, j)}$ Finally, since the coupons for different Directions and at different Locations are obtained from different plates, the measurements are considered as independent, and the likelihood function of Eq. (22) thus reads

$$
\pi\left(\hat{E}_{c} \mid\left[E_{0}, a_{r}\right]\right)=\prod_{i=1}^{n_{\text {pos }}} \prod_{j=1}^{(i)} \prod_{k=1}^{(i)} \pi_{n^{(i, j)}} n_{\text {sample }}^{(i, j)}\left(E_{c}^{(i, j, k)}-E_{\mathrm{MFH}}^{(i, j)}\left(\left[E_{0}, a_{r}\right]\right)\right] .
$$

Using Eq. (24) and Eq. (28), Bayes' formula (22) thus reads

$$
\pi_{\text {post }}\left(\left[E_{0}, a_{r}\right] \mid \hat{E}_{c}\right) \propto \prod_{i=1}^{n_{\text {pos }}} \prod_{j=1}^{n_{\text {dir }}^{(i)}} \prod_{k=1}^{n_{\text {sample }}^{(i, j)}} \pi_{n^{(i, j)}}\left[E_{c}^{(i, j, k)}-E_{\mathrm{MFH}}^{(i, j)}\left(\left[E_{0}, a_{r}\right]\right)\right] \pi_{\text {prior }}\left(\left[E_{0}, a_{r}\right]\right) .
$$

What remains to be defined is the set of noise functions $\pi_{n^{(i, j)}}$. A Gaussian function is selected as in several references [24-26, 28-31]:

$$
\pi_{n^{(i, j)}}(y)=\mathcal{N}_{0, \sigma_{E_{c}^{(i, j)}}^{2}}(y), \text { with } \mathcal{N}_{\mu, \sigma^{2}}(y)=\frac{1}{\sigma \sqrt{2 \pi}} \exp \left[-\frac{1}{2}\left(\frac{y-\mu}{\sigma}\right)^{2}\right] .
$$


The standard deviation is obtained from the experimental measurements as

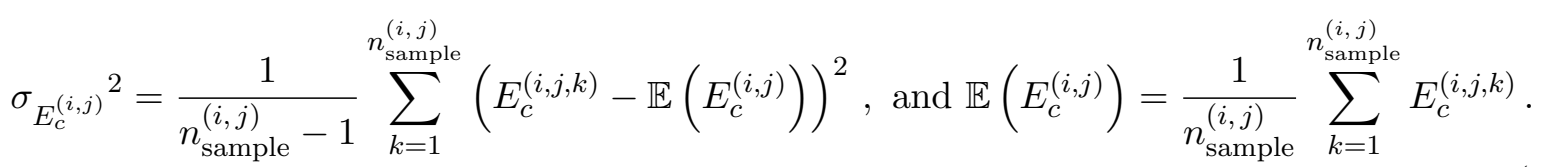

We note that a noise function has to be defined for each couple $(i, j)$ since the range of values of the measured Young's modulus is strongly affected by the loading direction.

\subsection{Distribution inference}

In the second approach, we assume that the matrix Young's modulus is heterogeneous in nature and is considered as a random variable. Physically the Young's modulus should, on the one hand, be strictly positive and, on the other hand, is known to be smaller than the glass fibers Young's modulus. Besides, numerically, the MFH process is only defined when the matrix Young's modulus is strictly positive and is numerically stable only if the matrix Young's modulus is upper-bounded by several times the inclusion Young's modulus. Therefore, we consider the matrix Young's modulus as a random variable following a lower- and upper-bounded distribution, which is chosen in the paper as a Beta distribution

$$
E_{0} \sim \beta_{\alpha, \beta, a, b} \quad \text { with } \beta_{\alpha, \beta, a, b}(y)=\frac{(y-a)^{\alpha-1}(b-y)^{\beta-1}}{(b-a)^{\alpha+\beta-1} B(\alpha, \beta)},
$$

where $a$ and $b$ define the function support, and $B(\alpha, \beta)$ is the normalization constant.

Because the model parameter is now seen as a random variable, the MFH model is now probabilistic with

$$
E_{\mathrm{MFH}} \sim \pi_{E_{\mathrm{MFH}}}\left(E_{0} ; \psi, \mathrm{I}\left(a_{r}\right), v_{\mathrm{I}}\right) \text {, and } E_{0} \sim \beta_{\alpha, \beta, a, b}
$$

where the inclusions I in the different pseudo-grains and the fiber volume fraction $v_{\mathrm{I}}$ depend on the plate Location and where $\psi$ depends on the loading Direction. In this case, we assume that the effective aspect ratio has already been estimated, e.g. using the error-based framework. This way of proceeding is justified by the different nature of the two parameters: the effective aspect ratio is a model parameter resulting from the model assumption and can thus be inferred as a deterministic value, while the matrix Young's modulus exhibits some variability that ought to be captured.

Because of the probabilistic nature of the MFH model (33), it is not straightforward to evaluate the likelihood using directly the measurements on the composite coupons as the observation data. 
Indeed, from a sampling of the 4 parameters of the Beta distribution, $\boldsymbol{x}=[\alpha, \beta, a, b]$, to be inferred, one can only deduce a distribution of the matrix Young's modulus and not the Young's modulus of the composite response. This distribution should thus be sampled too in order to perform the 2-step MFH and to evaluate the likelihood as in Section 5.2. This process would thus require a double Monte Carlo sampling, e.g., involving repeated computations of the 2-Step MFH model, which would not be computationally affordable. Instead, we consider as observation data $y=\hat{E}_{0 c}$, the matrix Young's modulii obtained by inverting the MFH function from the experimental measurements: $E_{c} \rightarrow E_{0 c}$, following Eq. (20), leading to the observations $E_{0 c}^{(m)}$ where $(m)$ indexes all the $n_{\text {coupons }}$ coupons cut at different Locations $(i)$ and tested along the Direction $\psi^{(j)}$.

The values to be inferred are now the 4 parameters of the Beta distribution, $\boldsymbol{x}=[\alpha, \beta, a, b]$. Concerning the shape parameters $\alpha$ and $\beta$, since they should be strictly larger than one we consider prior Gamma-distributions (23) in which we introduce the lower bound 1. Besides, since we do not have other information, the shape and rate parameters of the prior Gamma-distributions are chosen such that their variance is large (20000) enough not to bias the BI process. The support bounds $a$ and $b$ should be strictly positive and the only other available information comes from the experimental measurements range reported in Table 1 and from the inverse identification conducted in Fig. 6. We thus also consider prior Gamma-distributions for the support bounds. Concerning $a$, we introduce the lower bound $0.1 \mathrm{GPa}$ in the prior Gamma-distribution in order to remain strictly positive, and consider the shape and rate parameters of the prior Gamma-distribution so that its expectation corresponds to $2 \mathrm{GPa}$, which is lower than the values obtained by the inverse identification conducted in Fig. 6. Concerning $b$, we introduce the lower bound 2 GPa in the prior Gamma-distribution since all the values are expected to be larger than that, and consider the shape and rate parameters of the prior Gamma-distribution so that its expectation corresponds to $5 \mathrm{GPa}$, which is larger than the (non-rejected) values obtained by the inverse identification conducted in Fig. 6. Since we do not have prior knowledge on the correlation, we consider the prior Gamma-distributions as uncorrelated and the global prior thus reads

$$
\pi_{\text {prior }}([\alpha, \beta, a, b])=\Gamma_{2,0.01,1,1} \times \Gamma_{2,0.01,1,1} \times \Gamma_{2,1.05,0.1 \mathrm{GPa}, 1 \mathrm{GPa}} \times \Gamma_{2,0.665,2 \mathrm{GPa}, 1 \mathrm{GPa}} .
$$

Since the coupons for different Directions and at different Locations are obtained from different Plates, the observations $y=E_{0 c}$ are considered as independent, and the likelihood function is 
directly obtained from Eq. (32), i.e.

$$
\pi\left(E_{0 c}^{(m)} \mid[\alpha, \beta, a, b]\right)=\beta_{\alpha, \beta, a, b}\left(E_{0 c}^{(m)}\right) .
$$

Finally, Bayes' formula (22) thus reads

$$
\pi_{\text {post }}\left([\alpha, \beta, a, b] \mid \hat{E}_{0 c}\right) \propto \prod_{m=1}^{n_{\text {coupons }}} \beta_{\alpha, \beta, a, b}\left(E_{0 c}^{(m)}\right) \pi_{\text {prior }}([\alpha, \beta, a, b]) .
$$

\section{Results}

In this section the two Bayesian inference approaches presented in Section 5.2 and in Section 5.3 are applied on the experimental measurements performed on the PA06-GF40 plates and described in Section 3 at the exception of measurements at Location \#6, which will be further used for validation purpose. In order to analyze the resulting distributions, we use the average value $\mathbb{E}(\boldsymbol{x} \mid \boldsymbol{y})$ and the maximum a-posteriori-probability (MAP) point, which is defined as

$$
\operatorname{MAP}_{\boldsymbol{x} \mid \boldsymbol{y}}=\arg \max _{\boldsymbol{x}} \pi_{\text {post }}(\boldsymbol{x} \mid \boldsymbol{y})
$$

We consider both the MAP point and the average value since the MAP point is not always characteristic of most of the majority of the distributions.

\subsection{Application of error-based inference}

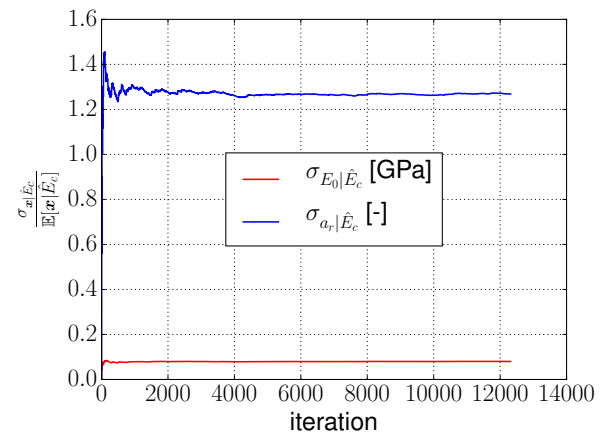

(a) $\sigma_{\boldsymbol{x} \mid \hat{E}_{c}}$

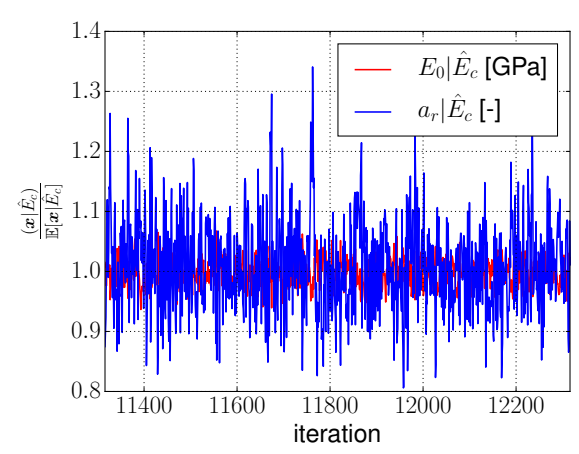

(b) $\operatorname{Trace}\left(\boldsymbol{x} \mid \hat{E}_{c}\right)$

Figure 7: Convergence study with respect to the MCMC iteration of the error-based BI when considering Location \#4: (a) Standard deviation of the posterior distribution, and (b) Trace of the inferred parameter.

We first apply Eq. (29) by considering successively coupons observations $E_{c}^{(m)}$ at Location \#3 for which 3 observations along the $0^{\circ}$-Direction exist, and at Location \#4 for which 9 observations exist along the different loading Directions. 


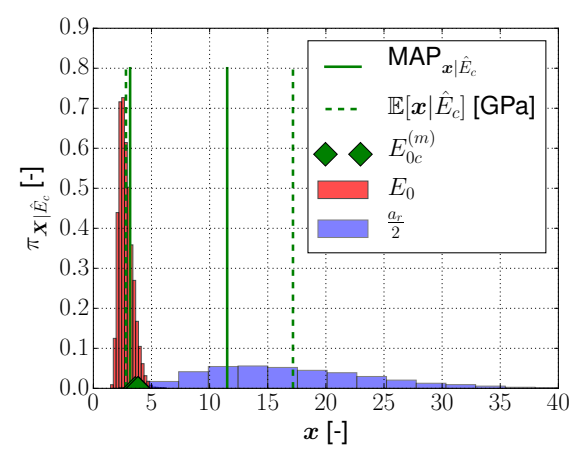

(a) Location $\# 3 ; \pi_{\boldsymbol{X} \mid \hat{E}_{c}}$

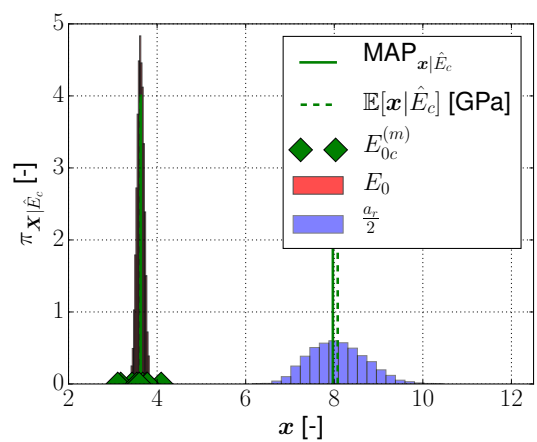

(c) Location \#4; $\pi_{\boldsymbol{X} \mid \hat{E}_{c}}$

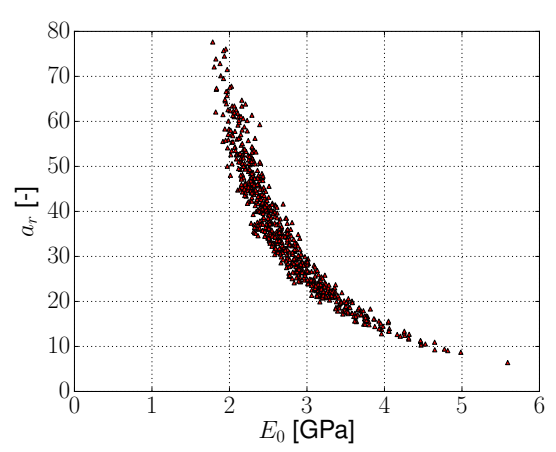

(b) Location \#3; $\left(\boldsymbol{x} \mid \hat{E}_{c}\right)$

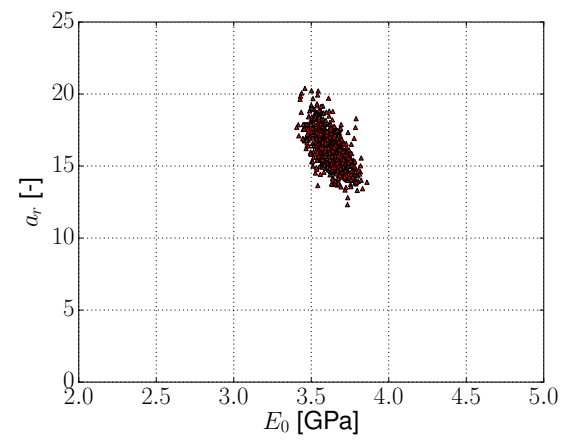

(d) Location \#4; $\left(\boldsymbol{x} \mid \hat{E}_{c}\right)$

Figure 8: Error-based BI, with (a) and (c) posterior marginal distributions $\pi_{\boldsymbol{X} \mid \hat{E}_{c}}$, and (b) and (d) randomly picked realizations of $\left(\boldsymbol{x} \mid \hat{E}_{c}\right)$ when considering the coupon measurements $\hat{E}_{c}$ from (a) Location \#3, and (b) Location \#4.

The convergence of the MCMC algorithm with respect to the iterations number, when considering the measurements from Location \#4, is studied in Fig. 7, in terms of the standard deviation of the posterior distribution, Fig. 7(a), and of the trace, i.e. the realizations in terms of the iteration number, in Fig. 7(b). The standard deviation is found to converge after a few thousands of iterations, and the trace has the typical fuzzy shape of a converged analysis.

The posterior marginal distributions $\pi_{\boldsymbol{x} \mid \hat{E}_{c}}$ are reported in Fig. 8, in which the matrix Young's modulus observations obtained using the change of variable (20), for an aspect ratio $a_{r}=16$, are also reported for illustration purpose (they are not used in the error-based BI). When considering only 3 observations along a unique Direction, it appears that the posterior distributions of the matrix Young's modulus and of the aspect ratio are wide enough so that the 3 experimental observations are embedded by the matrix Young's modulus distribution, see Fig. 8(a). The two distributions are strongly correlated, see Fig. 8(b) and an effective aspect ratio $a_{r}$ as a model parameter cannot be extracted. However, when considering observations from different loading 
directions, the set of realization points narrows, see Fig. 8(d). Nevertheless, as expected, when increasing the number of observations, the posterior becomes more peaky, see Fig. 8(c), and some observations are not included in the inferred distribution of the matrix Young's modulus. The MAP and average values are found to be the same since the distribution is (almost) Gaussian. Besides, when increasing the number of observations, the MAP converges to the "unique" solution of the maximum likelihood, which is also the mean value. In particular, an effective aspect ratio $a_{r}$ of 16 can be extracted from the MAP value.

As a conclusion, on the one hand, the aspect ratio $a_{r}$ can be inferred from the previous analysis and can be used in the subsequent analyzes, but on the other hand, the error-based inference cannot capture the heterogeneity of the matrix behavior observed in the experimental measurements.

\subsection{Application of distribution inference}

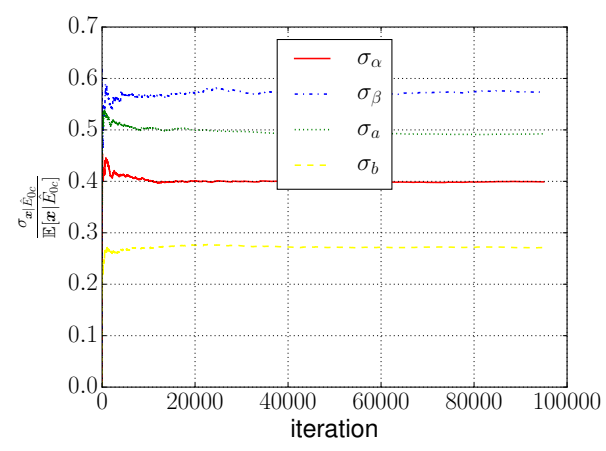

(a) $\sigma_{\boldsymbol{x} \mid \hat{E}_{0 c}}$

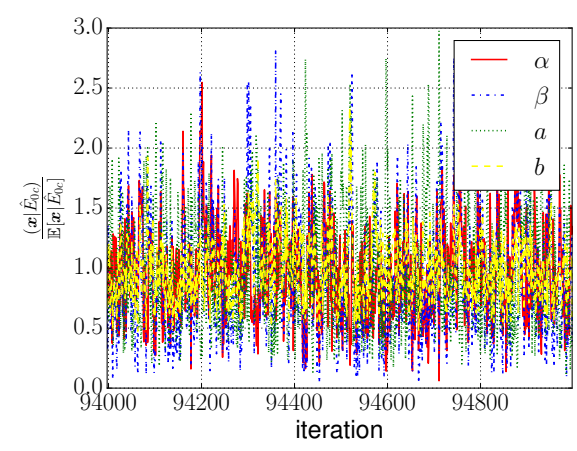

(b) $\operatorname{Trace}\left(\boldsymbol{x} \mid \hat{E}_{0 c}\right)$

Figure 9: Convergence study with respect to the MCMC iteration of the distribution-based BI when considering the Locations \#1-\#5 and all the possible loading Directions: (a) Standard deviation of the posterior distribution, and (b) Trace of the inferred parameters.

We now apply Eq. (36) considering successively a single Location with one loading Direction, and Locations \#1-\#5 with all the possible loading Directions altogether. For these analyzes, we consider an aspect ratio $a_{r}=16$, following the argumentation of the previous Section.

First the convergence of the MCMC algorithm, when considering the measurements from all the Locations and all the loading Directions, is assessed in Fig. 9. It can be seen that the standard deviation of the posterior distribution, Fig. 9(a), converges with the number of iterations. The trace, illustrated in Fig. 9(b), is typical from a converged analysis. 


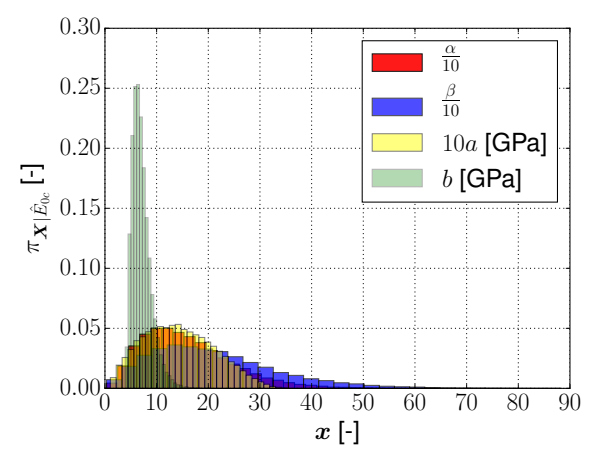

(a) Location \#3

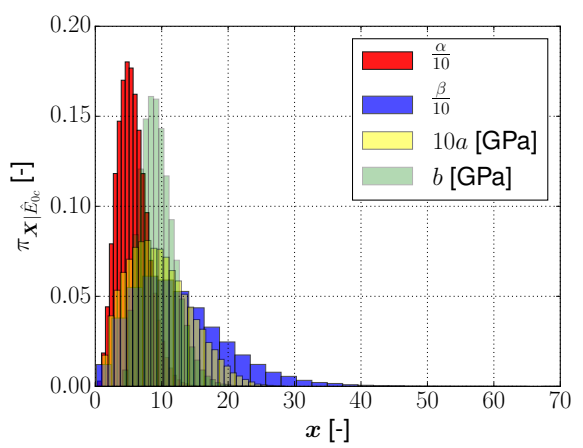

(b) Locations \#1-\#5

Figure 10: Posterior distributions $\pi_{\boldsymbol{x} \mid \hat{E}_{0 c}}$ of the distribution-based inferred parameters considering the measurements $\hat{E}_{0 c}$ from (a) Location \#3 only, and (b) Locations \#1-\#5.

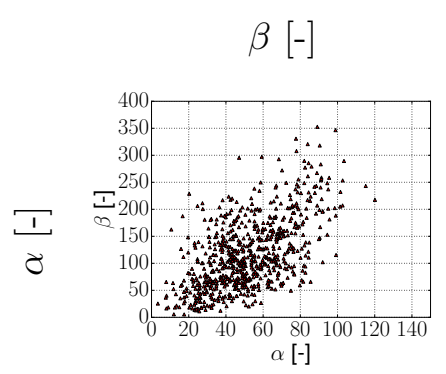

$\frac{1}{\infty}$

$\frac{\pi}{0}$
$a[\mathrm{GPa}]$
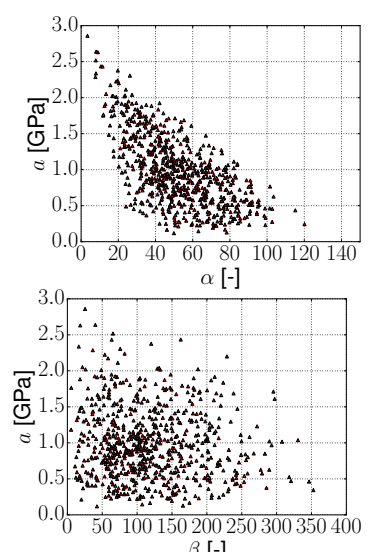

$\beta[-]$

\section{$b[\mathrm{GPa}]$}
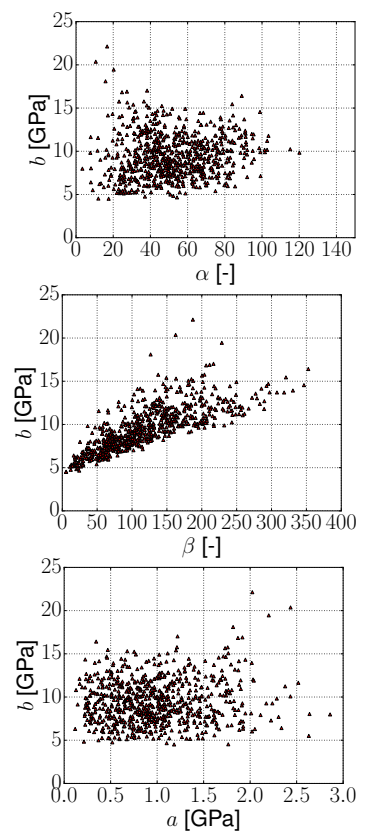

Figure 11: Randomly picked realizations of $\left(\boldsymbol{x} \mid \hat{E}_{0 c}\right)$ for the distribution-based BI method considering Locations \#1-\#5.

The posterior marginal distributions of the inferred parameters are illustrated in Fig. 10. When the bound $b$ of the support function tends to increase, this is compensated by high values of the exponent parameter $\beta$, in particular when less observations $E_{0 c}^{(m)}$ are considered, as in Fig. 10(a). This behavior is confirmed by Fig. 11, in which a few hundreds randomly picked realizations of $\left(\boldsymbol{x} \mid \hat{E}_{0 c}\right)$ are presented in order to study the dependency between the components: the main 
dependencies are observed between $\alpha$ and $\beta, \alpha$ and $a$, and $\beta$ and $b$.

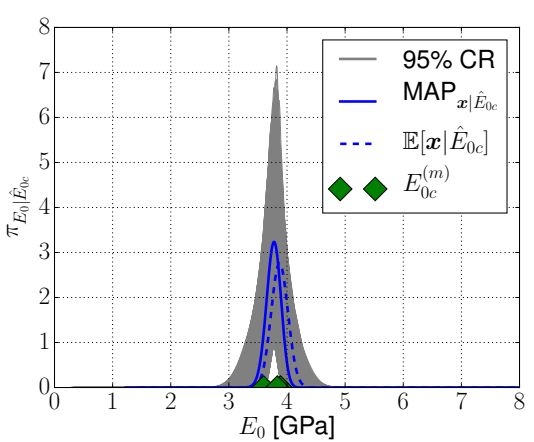

(a) Location \#3

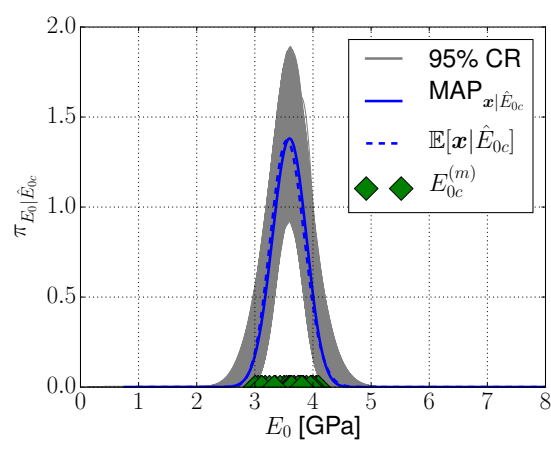

(b) Locations \#1-\#5

Figure 12: Posterior marginal distributions $\pi_{E_{0} \mid \hat{E}_{0 c}}$ of the distribution-based BI resulting matrix Young's modulus considering the measurements $\hat{E}_{0 c}$ from (a) Location \#4 only, and (b) Locations \#1-\#5.

Finally the resulting posterior distributions $\pi_{E_{0} \mid \hat{E}_{0 c}}$ of the matrix Young's modulus are reported in Fig. 12. Since this time we have inferred the parameters of the Young's modulus Beta distribution (32), the result of the BI is a set of Beta distributions, whose $95 \%$ Credible Region (CR) is depicted in Fig. 12. Besides, the distribution corresponding to the average value $\mathbb{E}\left(\boldsymbol{x} \mid \hat{E}_{0 c}\right)$ of the inferred parameters, and to the MAP (37) are also reported. It can be seen that the distributions embed all the experimental observations $E_{0 c}^{(m)}$ if one Location is considered, see Fig. 12(a), but also when Locations \#1-\#5 are considered, see Fig. 12(b). When only a limited number of observations is considered as in Fig. 12(a), the 95\% CR is much wider as compared to the case in which Locations \#1-\#5 are considered, see Fig. 12(b).

\subsection{Validation}

The parameters inferred using, first, the error-based approach in Section 6.2 , for which $E_{0}$ is directly inferred, and, then, the distribution-based approach in Section 6.2, for which the parameters of the $E_{0}$ distribution are inferred, are now used to predict the response uncertainty of the PA06-GF40 plate measurements at Location \#6. For the error-based BI, we use the parameters inferred considering Location \#4, while for the distribution-based BI we consider the Locations \#1-\#5.

The predictions are reported in Fig. 13, where the experimental measurements of Section 3 are also reported. On the one hand, the parameters inferred using the error-based BI do not lead to composite materials responses embedding the experimental measurements. On the other hand, the 


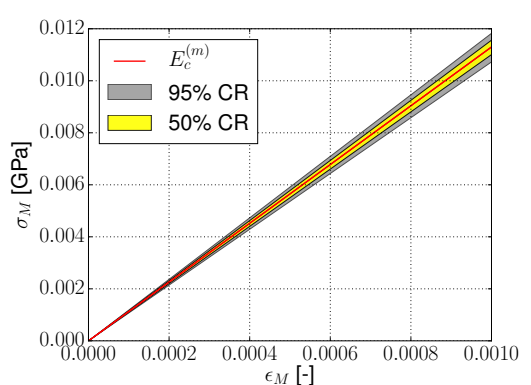

(a) $0^{\circ}$-loading; error-BI

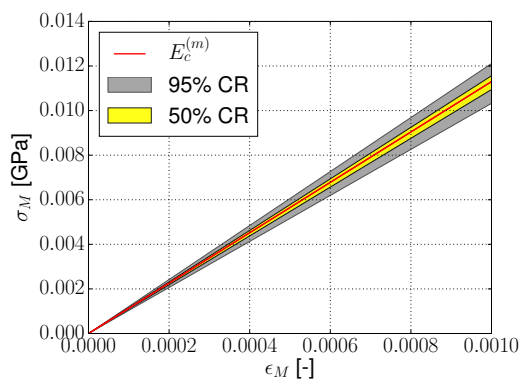

(d) $0^{\circ}$-loading; distribution-BI

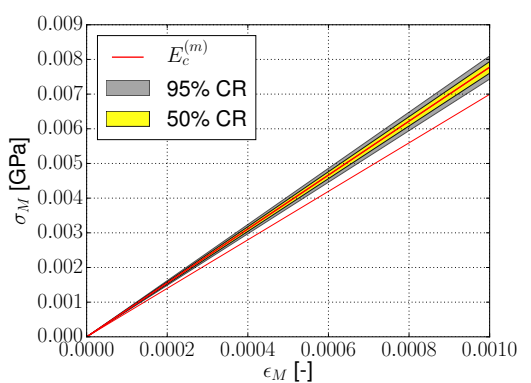

(b) $45^{\circ}$-loading; error-BI

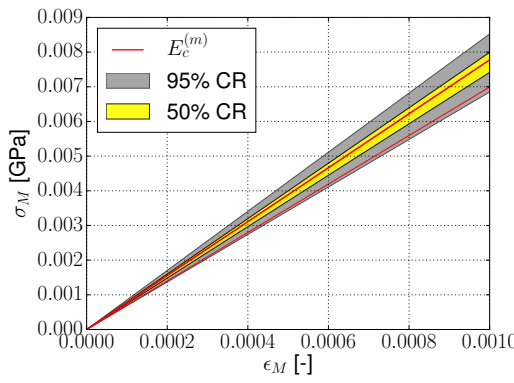

(e) $45^{\circ}$-loading; distribution-BI

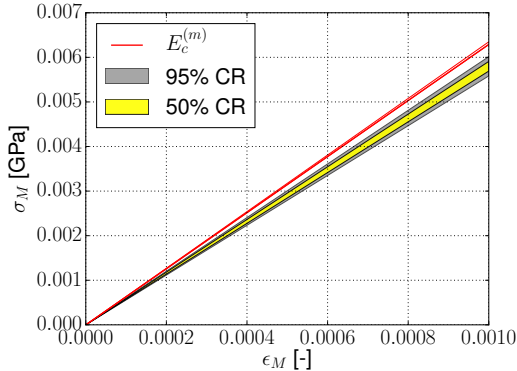

(c) $90^{\circ}$-loading; error-BI

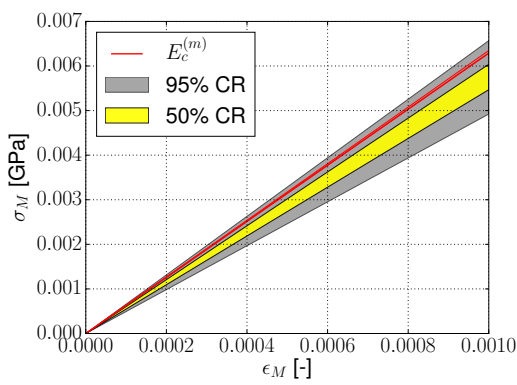

(f) $90^{\circ}$-loading; distribution-BI

Figure 13: Validation of the inferred parameters on the PA06-GF40 plate measurements at Location \#6, (a-c) using the error based approach, and (d-f) using the distribution based BI approach. Several loading Directions are considered.

distribution of the matrix Young's modulus obtained by the distribution-based BI predicts a $50 \%$ credible region of the composites responses which covers most of the experimental measurements and a $95 \%$ credible region, which covers them all.

\section{Conclusions}

We have shown in this paper that when modeling composite materials, although most the parameters of a multiscale model can be experimentally measured, on the one hand, some model parameters should still be defined, and, on the other hand, some physical parameters exhibit variability during experimental tests. Both families of parameters require a special identification process. To this end, two identification schemes based on Bayesian inference have been developed and compared: the first one considers a Gaussian noise function applied on the experimental measurements, and the second one assumes a normal distribution of the parameters to be identified, in which case the distribution parameters are inferred. While the first approach can directly uses the experimental observations of the composite responses to evaluate the likelihood function, the 
second approach requires first a change of variables to have as observations the corresponding parameters.

In this work, we have focused on the elastic response of Short Fibers Reinforced Polymers modeled using a MFH scheme. In particular, the effective aspect ratio of fiber populations, and the matrix Young's modulus have to be inferred.

When applying the two approaches on the experimental measurements, it is shown that the error-based approach narrows the posterior distribution when the number of observations increases. Although, this allows the model parameter, i.e. the effective aspect ratio, to be inferred, this makes the multiscale method unable to be used as a stochastic model since the uncertainties in the physical parameter, i.e. the matrix Young's modulus, cannot be predicted. On the contrary, the distribution-based approach is able to represent the matrix Young's modulus uncertainties and, as a result, the multiscale approach captures the stochastic behavior of the composite material experimentally observed. It is worth emphasizing that this stochasticity results directly from the identification process and not from a model.

In the future, it is intended to extend the distribution-based method to infer the matrix nonlinear model parameters in the elasto-(visco)-plastic case.

\section{Acknowledgment}

The research has been funded by the Walloon Region under the agreement no 1410246-STOMMMAC (CT-INT 2013-03-28) in the context of the M-ERA.NET Joint Call 2014. Leartikers research has been funded by the Gaitek 2015 programm of the Basque Government in the context of the MERA.NET Joint Call 2014

\section{References}

[1] P. Kanouté, D. Boso, J. Chaboche, B. Schrefler, Multiscale Methods for Composites: A Review, Archives of Computational Methods in Engineering 16 (2009) 31-75, ISSN 1134-3060, 10.1007/s11831-008-9028-8.

[2] L. Noels, L. Wu, L. Adam, Review of Homogenization Methods for Heterogeneous Materials, in: P. Ulrich, G. J. Schmitz (Eds.), Handbook of Software Solutions for ICME, chap. 6.1.1., Wiley-VCH, Weinheim, Germany, ISBN 978-3-527-33902-0, 433-441, 2016.

[3] K. Matous, M. G. Geers, V. G. Kouznetsova, A. Gillman, A review of predictive nonlinear theories for multiscale modeling of heterogeneous materials, Journal of Computational Physics 330 (Supplement C) (2017) 192 - 220 , ISSN 0021-9991, doi:10.1016/j.jcp.2016.10.070 
[4] J. D. Eshelby, The Determination of the Elastic Field of an Ellipsoidal Inclusion, and Related Problems, Proceedings of the Royal Society of London. Series A, Mathematical and Physical Sciences 241 (1226) (1957) pp. 376-396, ISSN 00804630 .

[5] T. Mori, K. Tanaka, Average stress in matrix and average elastic energy of materials with misfitting inclusions, Acta Metallurgica 21 (5) (1973) 571-574, cited By (since 1996) 1814.

[6] Y. Benveniste, A new approach to the application of Mori-Tanaka's theory in composite materials, Mechanics of Materials 6 (2) (1987) 147 - 157, ISSN 0167-6636, doi:DOI: 10.1016/0167-6636(87)90005-6.

[7] E. Kröner, Berechnung der elastischen Konstanten des Vielkristalls aus den Konstanten des Einkristalls, Zeitschrift für Physik A Hadrons and Nuclei 151 (1958) 504-518, ISSN 0939-7922, 10.1007/BF01337948.

[8] R. Hill, A self-consistent mechanics of composite materials, Journal of the Mechanics and Physics of Solids 13 (4) (1965) 213 - 222, ISSN 0022-5096, doi:DOI: 10.1016/0022-5096(65)90010-4.

[9] R. Hill, Continuum micro-mechanics of elastoplastic polycrystals, Journal of the Mechanics and Physics of Solids 13 (2) (1965) 89 - 101, ISSN 0022-5096, doi:DOI: 10.1016/0022-5096(65)90023-2.

[10] D. R. S. Talbot, J. R. Willis, Variational Principles for Inhomogeneous Non-linear Media, IMA Journal of Applied Mathematics 35 (1) (1985) 39-54, doi:10.1093/imamat/35.1.39.

[11] L. Wu, L. Noels, L. Adam, I. Doghri, A combined incremental-secant mean-field homogenization scheme with per-phase residual strains for elasto-plastic composites, International Journal of Plasticity 51 (2013) 80-102, doi:10.1016/j.ijplas.2013.06.006.

[12] C. W. Camacho, C. L. Tucker, S. Yalva c, R. L. McGee, Stiffness and thermal expansion predictions for hybrid short fiber composites, Polymer Composites 11 (4) (1990) 229-239, ISSN 1548-0569, doi:10.1002/pc.750110406, URL http://dx.doi.org/10.1002/pc.750110406.

[13] I. Doghri, L. Tinel, Micromechanical modeling and computation of elasto-plastic materials reinforced with distributed-orientation fibers, International Journal of Plasticity $21(10) \quad(2005) \quad 1919 \quad-$ 1940, ISSN 0749-6419, doi:10.1016/j.ijplas.2004.09.003, URL http://www. sciencedirect.com/science/article/pii/S0749641904001706.

[14] I. Doghri, L. Tinel, Micromechanics of inelastic composites with misaligned inclusions: Numerical treatment of orientation, Computer Methods in Applied Mechanics and Engineering 195 (13) (2006) 1387 - 1406, ISSN 0045-7825, doi:https://doi.org/10.1016/j.cma.2005.05.041, URL http://www.sciencedirect.com/science/article/pii/S0045782505002872, a Tribute to Thomas J.R. Hughes on the Occasion of his 60th Birthday.

[15] A. R. Clarke, C. N. Eberhardt, Microscopy Techniques for Materials Science, Woodhead Publishing Series in Electronic and Optical Materials, Woodhead Publishing, ISBN 978-1-85573-587-3, doi:https://doi.org/10.1533/9781855737501.frontmatter, URL http://www.sciencedirect.com/science/article/pii/B9781855735873500015, 2002.

[16] M. Vincent, T. Giroud, A. Clarke, C. Eberhardt, Description and modeling of fiber orientation in injection molding of fiber reinforced thermoplastics, Polymer $46 \quad(17)$ (2005) $6719 \quad-\quad 6725, \quad$ ISSN 0032-3861, doi:https://doi.org/10.1016/j.polymer.2005.05.026, URL http://www.sciencedirect.com/science/article/pii/S0032386105005598, polymer Blends, Composites 
and Hybrid Polymeric Materials.

[17] M. Gupta, K. K. Wang, Fiber orientation and mechanical properties of short-fiber-reinforced injectionmolded composites: Simulated and experimental results, Polymer Composites 14 (5) (1993) 367-382, doi: 10.1002/pc.750140503, URL https://onlinelibrary.wiley.com/doi/abs/10.1002/pc.750140503.

[18] H. Nouri, Modélisation et identification de lois de comportement avec endommagement en fatigue polycyclique de matriaux composite à matrice thermoplastique, Ph.D. thesis, Arts et Métiers ParisTech, Metz (France), URL https://pastel .archives-ouvertes.fr/pastel-00005669/document, 2009.

[19] C. Elster, G. Wübbeler, Bayesian regression versus application of least squares-an example, Metrologia 53 (1) (2016) S10, URL http://stacks.iop.org/0026-1394/53/i=1/a=S10.

[20] J. L. Beck, L. S. Katafygiotis, Updating Models and Their Uncertainties. I: Bayesian Statistical Framework, Journal of Engineering Mechanics 124 (1998) 455-461, URL https://ascelibrary.org/doi/pdf/10.1061.

[21] Progressing from least squares to Bayesian estimation, New York, USA, 1979.

[22] T. C. Lai, K. Ip, Parameter estimation of orthotropic plates by Bayesian sensitivity analysis, Composite Structures 34 (1) (1996) 29 - 42, ISSN 0263-8223, doi:https://doi.org/10.1016/0263-8223(95)00128-X, URL http://www.sciencedirect.com/science/article/pii/026382239500128X.

[23] F. Daghia, S. de Miranda, F. Ubertini, E. Viola, Estimation of elastic constants of thick laminated plates within a Bayesian framework, Composite Structures 80 (3) (2007) $461-473, \quad$ ISSN 0263-8223, doi:https://doi.org/10.1016/j.compstruct.2006.06.030, URL http://www. sciencedirect.com/science/article/pii/S0263822306002789.

[24] C. Gogu, W. Yin, R. Haftka, P. Ifju, J. Molimard, R. Le Riche, A. Vautrin, Bayesian Identification of Elastic Constants in Multi-Directional Laminate from Moiré Interferometry Displacement Fields, Experimental Mechanics 53 (4) (2013) 635-648, ISSN 1741-2765, doi:10.1007/s11340-012-9671-8, URL https://doi.org/10.1007/s11340-012-9671-8.

[25] T. Most, Identification of the parameters of complex constitutive models: Least squares minimization vs. Bayesian updating, in: D. Straub (Ed.), Reliability and Optimization of Structural Systems, CRC Press, 2010.

[26] H. Rappel, L. Beex, L. Noels, S. Bordas, Identifying elastoplastic parameters with Bayestheorem considering output error, input error and model uncertainty, Probabilistic Engineering Mechanics 55 (2019) 28-41, ISSN 0266-8920, doi:https://doi.org/10.1016/j.probengmech.2018.08.004, URL http://www. sciencedirect.com/science/article/pii/S0266892018300547.

[27] H. Rappel, L. Beex, J. Hale, L. Noels, S. Bordas, A tutorial on Bayesian inference to identify material parameters in solid mechanics, Archives of Computational Methods in Engineering ISSN 1134-3060.

[28] W. Hernandez, F. Borges, D. Castello, N. Roitman, C. Magluta, Bayesian Inference Applied on Model Calibration of a Fractional Derivative Viscoelastic Model, in: DINAME 2015 - Proceedings of the XVII International Symposium on Dynamic Problems of Mechanics, ISBN 1-4244-1306-0, URL https://ss14799. websiteseguro.com/swge5/PDF/DIN20150123_17225PDF, 2015.

[29] H. Rappel, L. A. A. Beex, S. P. A. Bordas, Bayesian inference to identify parameters in viscoelasticity, Mechanics of Time-Dependent Materials 22 (2) (2018) 221-258, ISSN 1573-2738, doi:10.1007/s11043-017-9361-0, URL https://doi.org/10.1007/s11043-017-9361-0. 
[30] S. Madireddy, B. Sista, K. Vemaganti, A Bayesian approach to selecting hyperelastic constitutive models of soft tissue, Computer Methods in Applied Mechanics and Engineering 291 (2015) $102-122$, ISSN 0045-7825, doi:https://doi.org/10.1016/j.cma.2015.03.012, URL http://www.sciencedirect.com/science/article/pii/S004578251500122X.

[31] P.-S. Koutsourelakis, A novel Bayesian strategy for the identification of spatially varying material properties and model validation: an application to static elastography, International Journal for Numerical Methods in Engineering 91 (3) (2012) 249-268, doi:10.1002/nme.4261, URL https://onlinelibrary.wiley.com/doi/abs/10.1002/nme.4261.

[32] R. G. Ghanem, A. Doostan, On the construction and analysis of stochastic models: Characterization and propagation of the errors associated with limited data, Journal of Computational Physics 217 (1) (2006) 63 - 81, ISSN 0021-9991, doi:https://doi.org/10.1016/j.jcp.2006.01.037, URL http://www.sciencedirect.com/science/article/pii/S0021999106000386, uncertainty Quantification in Simulation Science.

[33] M. Arnst, R. Ghanem, C. Soize, Identification of Bayesian posteriors for coefficients of chaos expansions, Journal of Computational Physics $229 \quad$ (9) $3134 \quad-\quad 3154, \quad$ ISSN 0021-9991, doi:https://doi.org/10.1016/j.jcp.2009.12.033, URL http://www.sciencedirect.com/science/article/pii/S0021999109007190.

[34] H. Rappel, L. Beex, Estimating fibres material parameter distributions from limited data with the help of Bayesian inference, European Journal of Mechanics - A/Solids 75 (2019) $169-196, \quad$ ISSN 0997-7538, doi:https://doi.org/10.1016/j.euromechsol.2019.01.001, URL http://www.sciencedirect.com/science/article/pii/S0997753818305916.

[35] B. Weber, B. Kenmeugne, J. Clement, J. Robert, Improvements of multiaxial fatigue criteria computation for a strong reduction of calculation duration, Computational Materials Science 15 (4) (1999) 381 - 399, ISSN 0927-0256, doi:https://doi.org/10.1016/S0927-0256(98)00129-3, URL http://www.sciencedirect.com/science/article/pii/S0927025698001293.

[36] Y. Benveniste, G. Dvorak, T. Chen, On diagonal and elastic symmetry of the approximate effective stiffness tensor of heterogeneous media, Journal of the Mechanics and Physics of Solids 39 (7) (1991) 927 - 946, ISSN 0022-5096, doi:https://doi.org/10.1016/0022-5096(91)90012-D, URL http://www.sciencedirect.com/science/article/pii/002250969190012D.

[37] E. Onat, F. Leckie, Representation of Mechanical Behavior in the Presence of Changing Internal Structure, Journal of Applied Mechanics 55 (1) (1988) 1-10, doi:10.1115/1.3173630.

[38] Akulon-General information on applications, processing and properties, Tech. Rep., DSM Engineering Plastics, 2003.

[39] J. A. M. Soons, I. de Baere, J. J. J. Dirckx, New Double Indentation Technique for Measurement of the Elasticity Modulus of Thin Objects, Experimental Mechanics 51 (1) (2011) 85-95, ISSN 1741-2765, doi:10.1007/s11340010-9340-8, URL https://doi.org/10.1007/s11340-010-9340-8.

[40] H. Haario, E. Saksman, J. Tamminen, Adaptive proposal distribution for random walk Metropolis algorithm, Computational Statistics 14 (3) (1999) 375-395, ISSN 0943-4062, doi:10.1007/s001800050022, URL 
https://doi.org/10.1007/s001800050022.

[41] W. Gilks, S. Richardson, D. Spiegelhalter, Markov Chain Monte Carlo in Practice, in: Chapman, Hall/CRC (Eds.), Chapman \& Hall/CRC Interdisciplinary Statistic, CRC press, Weinheim, Germany, ISBN $9780412055515,1995$.

[42] G. L. Hand, A theory of anisotropic fluids, Journal of Fluid Mechanics 13 (1) (1962) 33-46, doi: $10.1017 / \mathrm{S} 0022112062000476$.

[43] E. J. Hinch, L. G. Leal, Constitutive equations in suspension mechanics. Part 1. General formulation, Journal of Fluid Mechanics 71 (3) (1975) 481-495, doi:10.1017/S0022112075002698.

[44] S. G. Advani, C. L. Tucker III, Closure approximations for three-dimensional structure tensors, Journal of Rheology 34 (3) (1990) 367-386, doi:10.1122/1.550133.

\section{Data availability}

The raw/processed data required to reproduce these findings cannot be shared at this time as the data also forms part of an ongoing study.

\section{Appendix A. Closure approximation}

The orientation tensors denoted $\mathbb{A}^{l}(\boldsymbol{a})$ (linear closure) and $\mathbb{A}^{q}(\boldsymbol{a})$ (quadratic closure) are exact for randomly and aligned oriented inclusions, respectively. For other cases, only approximations as a function of the components of $\boldsymbol{a}$ do exist. Several approximations have been proposed in the literature:

- The linear approximation [42] is accurate for fiber populations oriented randomly (isotropic).

With this approximation, the fourth-order orientation tensor is written as

$$
\mathbb{A}^{l}(\boldsymbol{a})=\alpha_{l} \boldsymbol{I} \otimes \boldsymbol{I}+2\left(\alpha_{l}-\beta_{l}\right) \mathbb{I}+\beta_{l}[\boldsymbol{I} \otimes \boldsymbol{a}+\boldsymbol{a} \otimes \boldsymbol{I}]+2 \beta_{l}[\mathbb{I}(\boldsymbol{a}+\boldsymbol{I})-\mathbb{I}(\boldsymbol{a})],
$$

where $\boldsymbol{I}$ and $\mathbb{I}$ designate the symmetric second- and fourth-order identity tensors, respectively, and $I(\boldsymbol{a})_{i j k l}=\frac{1}{2}\left(a_{i k} a_{j l}+a_{i l} a_{j k}\right), \mathbb{I}=\mathbb{I}(\boldsymbol{I})$, and where

$$
\alpha_{l}=\frac{-1}{35} \text { and } \beta_{l}=\frac{1}{7} \text { in } 3 \mathrm{D} ; \text { and } \alpha_{l}=\frac{-1}{24} \text { and } \beta_{l}=\frac{1}{6} \text { in } 2 \mathrm{D} \text {. }
$$

- The quadratic approximation [43] is accurate when the fibers are aligned along the same direction. With this approximation, the fourth-order orientation tensor is written:

$$
\mathbb{A}^{q}(\boldsymbol{a})=\boldsymbol{a} \otimes \boldsymbol{a}
$$


- The hybrid approximation [44] combines the quadratic and linear closure approximations, with

$$
\mathbb{A}^{h}(\boldsymbol{a})=(1-f) \mathbb{A}^{l}(\boldsymbol{a})+f \mathbb{A}^{q}(\boldsymbol{a}), f=1-N_{h} \operatorname{det}(\boldsymbol{a}),
$$

where the number $N_{h}$ is $N_{h}=27$ in $3 \mathrm{D}$ and $N_{h}=4$ in $2 \mathrm{D}$. This hybrid approximation is accurate for the case of a population of oriented fibers in a single direction $(f=1)$ or for randomly oriented fiber populations, i.e. isotropic orientation $(f=0)$. Advani and Tucker [44] have shown that, overall, the hybrid approximations produce acceptable physical behaviors as compared to reality.

- The generalized hybrid approximation [14] fills the gap between the clearly 2D and 3D cases. To this end, the second-order orientation tensor $\boldsymbol{a}$ is first decomposed into its principal (ordered) components $a_{i}$ and eigenvectors $\boldsymbol{e}^{(i)}$, with $\boldsymbol{a}=\sum_{i} a_{i} \boldsymbol{e}^{(i)} \otimes \boldsymbol{e}^{(i)}$. We then work in the principal components space, designated by the notation $\bullet$ with

$$
\underline{\boldsymbol{a}}=\operatorname{diag}\left(a_{1}, a_{2}, a_{3}\right), \text { with } a_{1} \geq a_{2} \geq a_{3} \geq 0 \quad \& \quad a_{1}+a_{2}+a_{3}=1 .
$$

The normalized components of $\underline{\boldsymbol{a}}$ in the 1D, 2D and 3D cases are then used to evaluate the corresponding fourth-order orientation tensors. The 1D case considers the quadratic closure (A.3), i.e.

$$
\stackrel{1 \mathrm{D}}{\mathbb{A}}=\mathbb{A}^{q}(\stackrel{1 \mathrm{D}}{\underline{\boldsymbol{a}}}) \text { with } \stackrel{1 \mathrm{D}}{\boldsymbol{a}}=\operatorname{diag}(1,0,0) ;
$$

The 2D case considers the hybrid closure (A.4), i.e.

$$
\stackrel{2 \mathrm{D}}{\underline{\mathbb{A}}}=\mathbb{A}^{h}(\stackrel{2 \mathrm{D}}{\boldsymbol{a}}) \text { with } \stackrel{2 \mathrm{D}}{\underline{\boldsymbol{a}}}=\operatorname{diag}\left(\frac{a_{1}}{a_{1}+a_{2}}, \frac{a_{2}}{a_{1}+a_{2}}, 0\right) ; \text { and }
$$

The 3D case considers the hybrid closure (A.4), i.e.

$$
\stackrel{3 \mathrm{D}}{\mathbb{A}}=\mathbb{A}^{h}(\underline{3 \mathrm{D}}) \text { with } \underline{3 \mathrm{D}} \underline{\underline{\boldsymbol{a}}}=\underline{\boldsymbol{a}} .
$$

The fourth-order orientation tensor (in the $\boldsymbol{e}^{(i)}$ space) is then written as a convex combination of the closures associated with the normalized components of $\underline{\boldsymbol{a}}$ in the $1 \mathrm{D}, 2 \mathrm{D}$ and $3 \mathrm{D}$ cases as follows:

$$
\underline{\mathbb{A}}=\frac{a_{1}-a_{2}}{a_{1}} \underline{\mathbb{1}}+\frac{a_{2}-a_{3}}{a_{1}} \underline{\mathbb{D}}+\frac{a_{3}}{a_{1}} \underline{\mathbb{D}} .
$$

Finally, the generalized hybrid fourth-order orientation tensor $\mathbb{A}^{g h}$ is obtained by simple rotation to the original space

$$
A_{i j k l}^{g h}=e_{i}^{(m)} e_{j}^{(n)} e_{k}^{(o)} e_{l}^{(p)} \underline{A}_{m n o p} .
$$


In this work, we adopt the generalized hybrid fourth-order orientation tensor $\mathbb{A}^{g h}(\mathrm{~A} .10)$.

\section{Appendix B. Reconstruction of the ODF}

When unavailable, the ODF must be recovered from the orientation tensors $\boldsymbol{a}$ and $\mathbb{A}$. In this work we use the Onat and Leckie reconstruction method [37]:

$$
\pi_{\boldsymbol{P}}(\boldsymbol{p}) \approx \psi_{1}+\psi_{2}(\boldsymbol{a})^{\mathrm{dev}}:(\boldsymbol{p} \otimes \boldsymbol{p})^{\mathrm{dev}}+\psi_{3} \mathbb{B}(\boldsymbol{a}):: \mathbb{F}(\boldsymbol{p})
$$

with

$$
\mathbb{F}(\boldsymbol{p})=\mathbb{A}^{q}(\boldsymbol{p} \otimes \boldsymbol{p})-\mathbb{A}^{l}(\boldsymbol{p} \otimes \boldsymbol{p}), \text { and } \mathbb{B}(\boldsymbol{a})=\mathbb{A}^{g h}-\mathbb{A}^{l},
$$

where the (.) dev operator denotes the deviatoric part. The fourth-order orientation tensors $\mathbb{A}^{l}$ and $\mathbb{A}^{q}$ are computed using respectively Eqs. (A.1) and (A.3) as function of the components of either a or $(\mathbf{p} \otimes \mathbf{p})$, and the fourth-order orientation tensor $\mathbb{A}^{g h}$ is obtained using the generalized hybrid method (A.10). The constant values of $\psi_{1}, \psi_{2}$ and $\psi_{3}$ are given as

$$
\begin{gathered}
\psi_{1}=\frac{1}{4 \pi}, \psi_{2}=\frac{15}{8 \pi}, \psi_{3}=\frac{315}{32 \pi} \text { in } 3 \mathrm{D} ; \quad \text { and } \\
\psi_{1}=\frac{1}{2 \pi}, \psi_{2}=\frac{2}{\pi}, \psi_{3}=\frac{8}{\pi} \text { in } 2 \mathrm{D} .
\end{gathered}
$$

\section{Appendix C. Numerical averaging from the ODF}

From a practical point of view, we consider half of the unit sphere i.e. $(\theta, \phi) \in[0, \pi] \times[0, \pi]$. The angle $\theta$ is divided into constant increments $\Delta \theta=\frac{\pi}{N_{\theta}}$, defining $N_{\theta}$ spherical rings situated between the angles $(\theta-\Delta \theta)$ and $(\theta+\Delta \theta)$, and having the following area:

$$
S_{\theta}=\pi \int_{\left(\theta-\frac{\Delta \theta}{2}\right)}^{\left(\theta+\frac{\Delta \theta}{2}\right)} \sin (\xi) d \xi=2 \pi \sin (\theta) \sin \left(\frac{\Delta \theta}{2}\right) .
$$

In order to define iso-facets of (almost) constant area, for each of these rings, the number of iso-size facets is given by $N_{\phi}(\theta)=N_{\theta} \sin (\theta)$, leading to facet sizes

$$
S\left(\theta_{i}, \phi_{j}\right)=2 \Delta \theta \sin \left(\frac{\Delta \theta}{2}\right) .
$$

At each pole $\theta=0$ and $\theta=\pi$, there is a single facet of area

$$
S(\theta=0)=S(\theta=\pi)=\pi \int_{0}^{\left(\frac{\Delta \theta}{2}\right)} \sin (\xi) d \xi=\pi\left[1-\cos \left(\frac{\Delta \theta}{2}\right)\right]
$$


It is to be noted that in some cases, reconstructed probability functions have negative values. The recovered ODF, either in $2 \mathrm{D}$ or $3 \mathrm{D}$, is thus substituted by an analytical fit of its discrete values using a Gaussian function as suggested in [14], with

$$
\pi_{\boldsymbol{P}}(\phi) \approx \psi_{G}(\phi) \text { in } 2 \mathrm{D} ; \text { and } \pi_{\boldsymbol{P}}(\theta, \phi) \approx \psi_{G}(\theta) \psi_{G}(\phi) \text { in } 3 \mathrm{D}
$$

with $\psi_{G}$ a Gaussian function expressed as follows:

$$
\psi_{G}(\phi)=\frac{1}{\sigma_{\phi} \sqrt{2 \pi}} \exp \left[-\frac{1}{2}\left(\frac{\phi-\mathbb{E}(\phi)}{\sigma_{\phi}}\right)^{2}\right] ;
$$

where $\sigma_{\bullet}$ and $\mathbb{E}(\bullet)$ are the standard deviation and expectation of $\bullet$.

\section{Appendix D. Adaptive Metropolis algorithm}

We use the adaptive variant [40] of the metropolis algorithm, which is a specific case of the Metropolis-Hastings algorithm [41]. In this approach, the proposal distribution is symmetric and updated once every $n_{K}=1000$ samples.

A random walk is considered in the parameter space $\boldsymbol{x} \in \mathbb{R}^{n}$. Considering a proposition sample $\boldsymbol{x}^{(p)}$ drawn from a (symmetric) conditional distribution $q\left(\boldsymbol{x}^{(p)} \mid \boldsymbol{x}^{(i)}\right)$ with the current sample $\boldsymbol{x}^{(i)}$, depending on the ratio $\frac{\pi_{\text {post }}\left(\boldsymbol{x}^{(p)}\right)}{\pi_{\text {post }}\left(\boldsymbol{x}^{(i)}\right)}$ and on a random number $u$ drawn from a uniform distribution $\mathcal{U}_{0,1}$, with

$$
\mathcal{U}_{a, c}(y)= \begin{cases}\frac{1}{c-a} & \text { if } y \in[a, c] \\ 0 & \text { if } y \notin[a, c]\end{cases}
$$

the proposed sample either becomes the new current sample $\boldsymbol{x}^{(i)}$ or is rejected following the algorithm detailed in Table D.5.

Considering previous samples $\boldsymbol{x}^{(i)}$, the symmetric proposal distribution $q\left(\boldsymbol{x}^{(p)} \mid \boldsymbol{x}^{(i)}\right)$ is updated once every $n_{K}=1000$ samples, such that the proposition sample $\boldsymbol{x}^{(p)}$ is drawn following

$$
\boldsymbol{x}^{(p)} \sim \mathcal{N}_{\boldsymbol{x}^{(i)}, \gamma^{2} \boldsymbol{N}_{n_{K}}}
$$

where $\gamma$ determines the proposal distribution width and is usually taken as $\gamma=\frac{2.38}{\sqrt{n}}$. The matrix $\boldsymbol{N}_{n_{K}}$ of size $n \times n$ is obtained as the covariance matrix of the $n_{K}$ previous samples as

$$
\mathbf{N}_{n_{K}}=\frac{1}{n_{K}-1} \tilde{\boldsymbol{K}}^{T} \tilde{\boldsymbol{K}}
$$


where the $n_{K} \times n$ zero-mean matrix $\tilde{\boldsymbol{K}}$ collects $n_{K}$ previous samples indexed from $(j)$ to $\left(j+n_{K}\right)$ following

$$
\tilde{\boldsymbol{K}}=\left[\left[\boldsymbol{x}^{(j)}-\mathbb{E}(\boldsymbol{x})\right]^{T}, \ldots,\left[\boldsymbol{x}^{\left(j+n_{K}\right)}-\mathbb{E}(\boldsymbol{x})\right]^{T}\right]^{T} .
$$

In this last equation, the expectation $\mathbb{E}(\boldsymbol{x})=\frac{1}{n_{K}} \sum_{k=1}^{n_{K}} \boldsymbol{x}^{(j+k)}$ has been computed on the $n_{K}$ samples. Practically, the sample $\boldsymbol{x}^{(p)}$, Eq. (D.2), is obtained as

$$
\boldsymbol{x}^{(p)}=\boldsymbol{x}^{(i)}+\frac{\gamma}{\sqrt{n_{K}-1}} \tilde{\boldsymbol{K}}^{T} \boldsymbol{k}^{(p)} \quad \text { with } \boldsymbol{k}^{(p)} \sim \mathcal{N}_{0, \boldsymbol{I}_{n_{K}}},
$$

where $\boldsymbol{k}^{(p)}$ is drawn from the normal zero-mean distribution of unit covariance, $\boldsymbol{I}_{n_{K}}$ being the $n_{K} \times n_{K}$ identity matrix.

\section{Table D.5: Adaptive Metropolis algorithm}

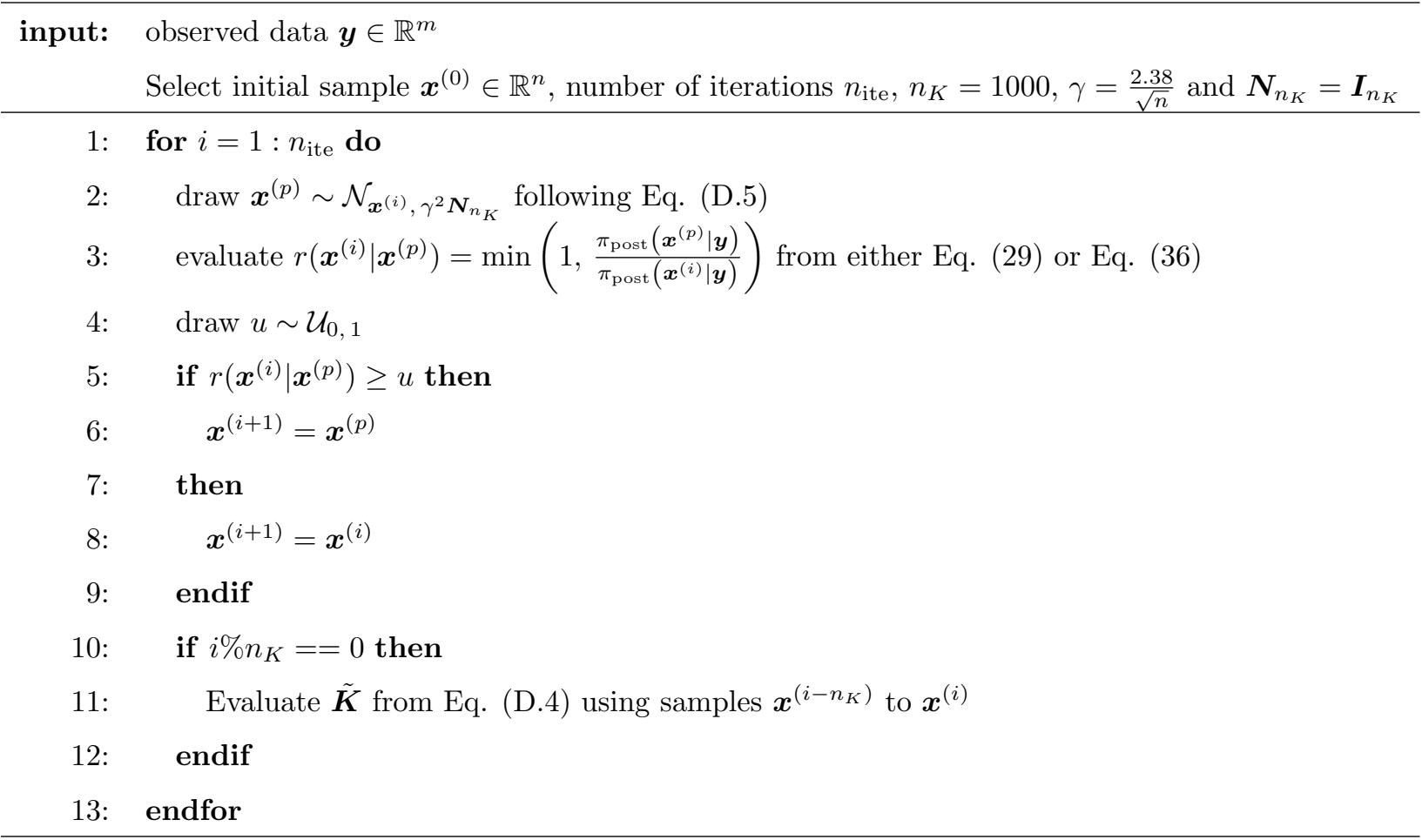

